\title{
Article
}

\section{The COMBS Survey - III. The Chemodynamical Origins of Metal-Poor Bulge Stars*}

Lucey, Madeline, Hawkins, Keith, Ness, Melissa, Nelson, Tyler, Debattista, Victor P, Luna, Alice, Bensby, Thomas, Freeman, Kenneth $\mathrm{C}$ and Kobayashi, Chiaki

Available at http://clok.uclan.ac.uk/39553/

Lucey, Madeline, Hawkins, Keith, Ness, Melissa, Nelson, Tyler, Debattista, Victor P ORCID: 0000-0001-7902-0116, Luna, Alice, Bensby, Thomas, Freeman, Kenneth C and Kobayashi, Chiaki (2021) The COMBS Survey - III. The Chemodynamical Origins of Metal-Poor Bulge Stars*. Monthly Notices of the Royal Astronomical Society, 509 (1). pp. 122-144. ISSN 0035-8711

It is advisable to refer to the publisher's version if you intend to cite from the work. http://dx.doi.org/10.1093/mnras/stab2878

For more information about UCLan's research in this area go to

http://www.uclan.ac.uk/researchgroups/ and search for <name of research Group>.

For information about Research generally at UCLan please go to http://www.uclan.ac.uk/research/

All outputs in CLoK are protected by Intellectual Property Rights law, including Copyright law. Copyright, IPR and Moral Rights for the works on this site are retained by the individual authors and/or other copyright owners. Terms and conditions for use of this material are defined in the policies page.

\section{CLoK}

Central Lancashire online Knowledge www.clok.uclan.ac.uk

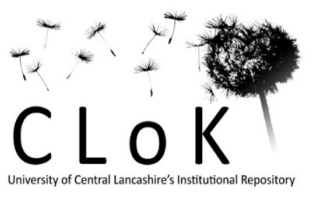




\title{
The COMBS Survey - III. The chemodynamical origins of metal-poor bulge stars
}

\author{
Madeline Lucey ${ }^{\circledR},{ }^{1 \star}$ Keith Hawkins ${ }^{\circledR 0},{ }^{1}$ Melissa Ness, ${ }^{2,3}$ Tyler Nelson, ${ }^{1}$ Victor P. Debattista ${ }^{\circledR}, 4$ \\ Alice Luna, ${ }^{1,5}$ Thomas Bensby, ${ }^{6}$ Kenneth C. Freeman ${ }^{7}$ and Chiaki Kobayashi ${ }^{\circledR 8}$ \\ ${ }^{1}$ Department of Astronomy, The University of Texas at Austin, 2515 Speedway Boulevard, Austin, TX 78712, USA \\ ${ }^{2}$ Center for Computational Astrophysics, Flatiron Institute, 1625 th Avenue, New York, NY 10010, USA \\ ${ }^{3}$ Department of Astronomy, Columbia University, 550 W 120th Street, New York, NY 10027, USA \\ ${ }^{4}$ Jeremiah Horrocks Institute, University of Central Lancashire, Preston PR1 2HE, UK \\ ${ }^{5}$ Department of Astronomy and Astrophysics, University of Chicago, Chicago, IL 60637, USA \\ ${ }^{6}$ Lund Observatory, Department of Astronomy and Theoretical Physics, Box 43, SE-221 00 Lund, Sweden \\ ${ }^{7}$ Research School of Astronomy and Astrophysics, The Australian National University, Canberra, ACT 2611, Australia \\ ${ }^{8}$ Centre for Astrophysics Research, Department of Physics, Astronomy and Mathematics, University of Hertfordshire, Hatfield AL10 9AB, UK
}

Accepted 2021 October 1. Received 2021 October 1; in original form 2021 July 6

\begin{abstract}
The characteristics of the stellar populations in the Galactic bulge inform and constrain the Milky Way's formation and evolution. The metal-poor population is particularly important in light of cosmological simulations, which predict that some of the oldest stars in the Galaxy now reside in its centre. The metal-poor bulge appears to consist of multiple stellar populations that require dynamical analyses to disentangle. In this work, we undertake a detailed chemodynamical study of the metal-poor stars in the inner Galaxy. Using $R \sim 20000$ VLT/GIRAFFE spectra of 319 metal-poor $(-2.55 \mathrm{dex} \leq[\mathrm{Fe} / \mathrm{H}] \leq 0.83 \mathrm{dex}$, with $\overline{[\mathrm{Fe} / \mathrm{H}]}=$ $-0.84 \mathrm{dex}$ ) stars, we perform stellar parameter analysis and report 12 elemental abundances (C, $\mathrm{Na}, \mathrm{Mg}, \mathrm{Al}, \mathrm{Si}, \mathrm{Ca}, \mathrm{Sc}, \mathrm{Ti}, \mathrm{Cr}$, $\mathrm{Mn}, \mathrm{Zn}, \mathrm{Ba}$, and $\mathrm{Ce}$ ) with precisions of $\approx 0.10 \mathrm{dex}$. Based on kinematic and spatial properties, we categorize the stars into four groups, associated with the following Galactic structures: the inner bulge, the outer bulge, the halo, and the disc. We find evidence that the inner and outer bulge population is more chemically complex (i.e. higher chemical dimensionality and less correlated abundances) than the halo population. This result suggests that the older bulge population was enriched by a larger diversity of nucleosynthetic events. We also find one inner bulge star with a $[\mathrm{Ca} / \mathrm{Mg}]$ ratio consistent with theoretical pair-instability supernova yields and two stars that have chemistry consistent with globular cluster stars.
\end{abstract}

Key words: stars: abundances - stars: Population II - Galaxy: bulge-Galaxy: evolution.

\section{INTRODUCTION}

The goal of Galactic archaeology is to understand the Milky Way's (MW) formation and evolution through the chemodynamical properties of its stars. Using observations (Ortolani et al. 1995; Kuijken \& Rich 2002; Zoccali et al. 2003; Brown et al. 2010; Clarkson et al. 2011; Valenti et al. 2013; Calamida et al. 2014; Howes et al. 2014) and simulations (Tumlinson 2010; Kobayashi \& Nakasato 2011; Starkenburg et al. 2017a; El-Badry et al. 2018b), the bulge of the MW has been shown to contain many of the oldest stars in our Galaxy. Studies of the chemodynamics of these old stars can reveal new insights into the formation and early chemical evolution of the MW.

The bulge is a complex Galactic component, with many overlapping stellar populations. Spectroscopic studies of the stars in the bulge have revealed a metallicity distribution function (MDF) with multiple components. Specifically, the Abundances and Radial velocity Galactic Origins Survey (ARGOS; Freeman et al. 2013)

^E-mail: m_lucey@utexas.edu found that the MDF of the bulge has five components (Ness et al. 2013a). The two most metal-rich components, which are associated with the bulge, peak at $[\mathrm{Fe} / \mathrm{H}]=+0.12$ and -0.25 dex. The other three components, which peak at $[\mathrm{Fe} / \mathrm{H}]=-0.70,-1.18$, and -1.70 dex, they associate with the thin disc, thick disc, and halo components of the MW, respectively. However, it is important to note that the metal-rich components dominate with only 5 per cent of bulge stars having $[\mathrm{Fe} / \mathrm{H}]<-1$ dex (Ness \& Freeman 2016). Although many studies have found similar results (e.g. Zoccali et al. 2008, 2017; Johnson et al. 2013; Rojas-Arriagada et al. 2014, 2017; Duong et al. 2019a), Johnson et al. (2020) argue that the multimodal MDF is only valid for the outer bulge and that inside a Galactic latitude of $(b) \sim$ $6^{\circ}$ the MDF is consistent with a closed box model (a single peak with a long metal-poor tail). However, Bensby et al. (2013, 2017) found strikingly similar results to Ness et al. (2013a) using bulge micro-lensed dwarf stars within $-6^{\circ}<b<-2^{\circ}$.

The discovery of metallicity-dependent structure and kinematics in the bulge provides further evidence of multiple stellar populations (Ness et al. 2013a,b). Today, it is generally accepted that the majority of the mass in the bulge participates in a boxy/peanut-shaped $(\mathrm{B} / \mathrm{P})$ bulge (Howard et al. 2009; Shen et al. 2010; Ness et al. 2013b; 
Debattista et al. 2017). A B/P bulge is a rotation-supported structure, which is the result of secular disc and bar evolution (Combes \& Sanders 1981; Combes et al. 1990; Raha et al. 1991; Merritt \& Sellwood 1994; Quillen 2002; Bureau \& Athanassoula 2005; Debattista et al. 2006; Quillen et al. 2014; Sellwood \& Gerhard 2020). However, it is also suggested that the MW may host a less massive metal-poor classical bulge component (Babusiaux et al. 2010; Hill et al. 2011; Zoccali et al. 2014), which is a spheroidal, pressuresupported structure formed by hierarchical accretion (Kauffmann, White \& Guiderdoni 1993; Kobayashi \& Nakasato 2011; Guedes et al. 2013). Evidence of a metal-poor classical bulge has been found in studies of the kinematics of bulge stars as a function of metallicity. Specifically, metal-poor stars in the bulge rotate slower and have a higher velocity dispersion than the metal-rich stars (Ness et al. 2013b; Kunder et al. 2016; Arentsen et al. 2020a). However, Debattista et al. (2017) demonstrated that these observations may be the result of an overlapping halo population rather than a classical bulge. In fact, Kunder et al. (2020) found that 25 per cent of the RR Lyrae stars currently in the bulge are actually halo interlopers. Similarly, Lucey et al. (2020) found that about 50 per cent of their sample of metal-poor giants are halo interlopers and that the fraction of interlopers increases with decreasing metallicity. When they removed the halo interlopers from the sample, Lucey et al. (2020) found that the velocity dispersion decreased and there was no evidence of a classical bulge component in the kinematics.

With the advent of metallicity-sensitive photometric surveys such as the SkyMapper (Casagrande et al. 2019) and Pristine (Starkenburg et al. 2017b) surveys, there is great potential to target and study the metal-poor stars in the Galactic bulge. These metal-poor stars are especially exciting because previous work on old stars has focused on the Galactic halo, where the majority of stars are metal poor (e.g. Frebel et al. 2006; Norris et al. 2007; Christlieb et al. 2008; Keller et al. 2014). Simulations now indicate that targeting metalpoor stars in the bulge is most conducive to the discovery of ancient stars. For example, simulations predict that if Population III stars exist in our Galaxy, they are most likely to be found in the bulge (White \& Springel 2000; Brook et al. 2007; Diemand et al. 2008). Furthermore, simulations predict that stars of a given metallicity are more likely to be older if they are found closer to the Galactic Centre (Salvadori et al. 2010; Tumlinson 2010; Kobayashi \& Nakasato 2011). Specifically, metal-poor bulge stars are ancient in that they formed before $z>5$ and are older than 12 Gyr (Kobayashi \& Nakasato 2011).

The chemistry of ancient stars is of special interest, given that they are thought to be primarily enriched by Population III stars. Therefore, their chemistry can provide insight into the properties of Population III stars and the early Universe in which they formed. Several studies have found that a significant fraction of Population III stars would explode as pair-instability supernovae (PISNe) given that simulations of metal-free star formation yield a top-heavy initial mass function (IMF; Tumlinson 2006; Heger \& Woosley 2010; Bromm 2013). Results of simulated yields from PISNe predict that a star that is 90 per cent enriched by a PISN would have $[\mathrm{Fe} / \mathrm{H}] \approx-2.5 \mathrm{dex}$ (Karlsson, Johnson \& Bromm 2008) and would contain barely any elements heavier than Fe (Karlsson et al. 2008; Kobayashi, Tominaga \& Nomoto 2011b; Takahashi, Yoshida \& Umeda 2018). Recently, Takahashi et al. (2018) found that the two most discriminatory abundance ratios that indicate enrichment from PISNe are $[\mathrm{Na} / \mathrm{Mg}]$ $\approx-1.5 \mathrm{dex}$ and $[\mathrm{Ca} / \mathrm{Mg}] \approx 0.5-1.3 \mathrm{dex}$. Excluding PISNe (i.e. if the IMF is truncated at $<140 \mathrm{M}_{\odot}$ ), ancient stars are expected to have higher levels of $\alpha$-element enhancement than typical MW stars due to the top-heavy IMF of Population III stars and the mass-dependent yields of Type II supernovae (Heger \& Woosley 2010; Tumlinson 2010; Bromm 2013). Another important chemical signature of ancient stars is lower copper $(\mathrm{Cu})$, manganese $(\mathrm{Mn})$, sodium $(\mathrm{Na})$, and aluminium $(\mathrm{Al})$ abundances with respect to typical MW stars given the metallicity dependence of these yields in Type II supernovae (Kobayashi \& Nakasato 2011).

Recently, there have been many spectroscopic surveys targeting the metal-poor stars in the bulge (e.g. Howes et al. 2014, 2015, 2016; Duong et al. 2019a,b; Lucey et al. 2019; Arentsen et al. 2020b). The first instalment of the Chemical Origins of Metal-poor Bulge Stars (hereafter COMBS I) studied the detailed chemistry of 26 metal-poor bulge stars (Lucey et al. 2019). One of the major results from this work was the discovery of higher levels of calcium enhancement in the bulge compared to Galactic halo stars of similar metallicity. Furthermore, COMBS I found lower scatter in many elemental abundances for very metal poor bulge stars compared to halo stars. The HERMES Bulge Survey (HERBS; Duong et al. 2019a) and Fulbright, McWilliam \& Rich (2007) found similar results with respect to higher levels of $\mathrm{Ca}$ enhancement and lower scatter for their sample of metal-poor stars. Further differences between metal-poor bulge stars and halo stars include the rate of carbon (C) and neutron process enhancements. C-enhanced metal-poor (CEMP) stars occur at a rate of 15-20 per cent among halo stars with $[\mathrm{Fe} / \mathrm{H}]<-2$ dex (Yong et al. 2013). However, in the bulge, the rate of CEMP stars is estimated at $\sim 6$ per cent for the same metallicity range (Arentsen et al. 2021). Furthermore, neutron-capture element-enhanced stars are rarely observed in bulge spectroscopic surveys (Johnson et al. 2012; Duong et al. 2019b; Koch et al. 2019; Lucey et al. 2019).

It is important to note, however, that $\sim 25-50$ per cent of metal-poor stars in the bulge are actually halo interlopers (Kunder et al. 2020; Lucey et al. 2020). Therefore, it is unclear whether these chemistry results simply apply to the Galactic halo in the inner Galaxy, to the Galactic bulge, or both. Consequently, dynamical analysis is essential to study these populations separately. Given results from simulations (Tumlinson 2010), metal-poor stars on tightly bound orbits are expected to have formed as early as $z \sim 20$ while stars on loosely bound orbits only form as early as $z \sim 10-13$. This is because stars on loosely bound orbits, which are accreted more recently, originate from small dark matter haloes that form later than the most massive main progenitors (Tumlinson 2010). This is consistent with recent simulation results demonstrating that the majority of stars within $2 \mathrm{kpc}$ of the Galactic Centre formed in the most massive main progenitor of the MW (Santistevan et al. 2020). Therefore, we expect that stars confined to the inner bulge region are more ancient than loosely bound halo stars. However, it is essential to combine chemical and dynamical information to test this prediction and compare these populations in detail.

In this work, we aim to determine the origins of the metal-poor stars in the Galactic bulge through chemodynamical analysis. Specifically, we will test predictions from simulations that the metal-poor bulge stars are ancient and search for signatures of PISNe. To accomplish this, we present the stellar parameters and elemental abundances for a sample of 319 stars selected to be metal-poor bulge stars using SkyMapper photometry. We combine this analysis with dynamical results from the second instalment of the COMBS survey (Lucey et al. 2020, hereafter COMBS II) for a full chemodynamical picture. In Section 2, we present the VLT/GIRAFFE observations and data reduction method. The stellar parameter and elemental abundance analysis are described in Sections 3 and 4, respectively. We perform a comparison between our analysis, the ARGOS survey, and the HERBS survey in Section 5. We present our MDF and elemental abundance results in Sections 6 and 7, respectively. We separate our 
population into four dynamical groups and compare their chemistry in Section 8. We discuss chemical signatures of PISNe in Section 9 and possible globular cluster origins for our stars in Section 10. Last, we present our final conclusions in Section 11.

\section{DATA}

Given the high levels of extinction and primarily metal-rich population, obtaining large spectroscopic samples of metal-poor stars in the Galactic bulge has historically been difficult. With the advent of metallicity-sensitive photometric surveys, like the SkyMapper (Wolf et al. 2018) and Pristine (Starkenburg et al. 2017b) surveys, it is now possible to target and observe these rare stars in large numbers. In this work, we use SkyMapper photometry and ARGOS spectra (Freeman et al. 2013) to select metal-poor giants for spectroscopic follow-up. For further information on the target selection, we refer the reader to Section 2 of COMBS I.

The observations presented in this work are from the FLAMES spectrograph (Pasquini et al. 2002) on the European Southern Observatory's (ESO) Very Large Telescope (VLT). The FLAMES instrument is fibre fed with fibres going to both the UVES and GIRAFFE spectrographs. Therefore, observations with both spectrographs can be simultaneously obtained. For the COMBS survey, we observed 555 stars with the GIRAFFE spectrograph along with 40 stars with the UVES spectrograph. For the UVES spectra, we used the RED580 set-up that has a resolution $R=\lambda / \Delta \lambda$ $\approx 47000$ and a wavelength coverage 4726-6835 $\AA$. The stellar parameters and elemental abundances of the UVES spectra have already been published in COMBS I. In this work, we present the stellar parameter and chemical abundance analysis of the GIRAFFE spectra.

\subsection{Medium-resolution GIRAFFE spectra}

For the GIRAFFE spectra, we use the HR06 and HR21 set-ups. The HR06 set-up has a resolution $R \approx 24300$ and a wavelength coverage 4538-4759 $\AA$, while the HR21 set-up has a resolution $R \approx 18000$ and a wavelength coverage 8484-9001 $\AA$. The HR21 spectra contain the Calcium II near-infrared triplet (CaT), which is useful for determining accurate radial velocities. The HR06 spectra contain many metal lines including iron $(\mathrm{Fe})$ lines for constraining the metallicity and even a barium (Ba) line (4554 $\AA$ ) in order to measure the s-process abundance. It also contains a number of $\mathrm{C}_{2}$ Swan band features with band heads at approximately 4715, 4722, 4737, and $4745 \AA$. Therefore, we can also determine if a star is a CEMP star with or without s-process enhancement (CEMP-s or CEMP-no).

As these spectra were used to perform kinematic analysis in COMBS II, the full description of the reduction process can be found in section 2.2 of that paper. In short, we use the EsoReflex ${ }^{1}$ workflow to perform the bias and flat-field subtraction, along with fibre-tofibre corrections, cosmic ray cleaning, wavelength calibration, and extraction. We then use IRAF to perform sky subtraction. Last, we use iSpec (Blanco-Cuaresma et al. 2014) to radial velocity (RV) correct, coadd, and normalize the spectra. During the RV determination, we find two possible spectroscopic binary stars (labelled as 6406.0 and 6400.2 in the ESO Phase 3 Data Products archive ${ }^{2}$ ) that both

\footnotetext{
${ }^{1}$ https://www.eso.org/sci/software/esoreflex/
}

${ }^{2}$ http://archive.eso.org/wdb/wdb/adp/phase3_spectral/form have two significant peaks (peak probability $>0.5$ ) in the crosscorrelation function. As unresolved spectroscopic binaries can lead to systematic biases in stellar parameters (e.g. El-Badry et al. 2018a), we do not perform stellar parameter analysis on these stars.

We estimate the signal-to-noise ratio (SNR) using the flux uncertainty estimates from the EsoReflex pipeline that are propagated through the reduction process. We do not use any individual spectra with SNR $<10$ pixel $^{-1}$. Out of 555, there are 545 stars with HR2 1 spectra with SNR $>10$ pixel $^{-1}$ and only 389 stars with both HR06 and HR21 spectra having SNR $>10$ pixel $^{-1}$. It is expected that the HR06 spectra have lower SNR on average compared to the HR21 spectra since they are bluer and therefore more impacted by the high levels of extinction towards the Galactic Centre. In this work, we analyse only stars that have both HR06 and HR21 spectra for consistency. Therefore, after removing the two possible binary stars, there are a total of 387 stars for which we perform stellar parameter analysis.

\section{STELlar Parameter anAlysis}

Given the wavelength coverage and resolution of our spectra, there are not enough clean Fe I and Fe II lines to perform the standard Fe-excitation-ionization balance technique to determine the stellar parameters. Therefore, in this work we use a full-spectrum $\chi^{2}$ fitting technique to determine the effective temperature $\left(T_{\text {eff }}\right)$, surface gravity $(\log g)$, metallicity $([\mathrm{M} / \mathrm{H}])$, and rotational velocity $(V \sin i)$.

The model spectra, which we use to compare to the observed spectra, are synthesized using Spectroscopy Made Easy (SME) v574 (Valenti \& Piskunov 1996; Piskunov \& Valenti 2017). To synthesize spectra, we utilize the $1 \mathrm{D}$, local thermodynamic equilibrium (LTE) MARCS model atmosphere grid (Gustafsson et al. 2008) and the fifth version of the Gaia-ESO atomic line list that includes hyperfine structure (Heiter et al. 2020). In addition, we use solar abundances from Grevesse, Asplund \& Sauval (2007). We incorporate non-LTE (NLTE) line formation for a number of elements using grids of departure coefficients. We use all grids available with SME v574 that includes lithium (Li; Lind, Asplund \& Barklem 2009), oxygen (O; Amarsi et al. 2016), $\mathrm{Na}$ (Lind et al. 2011), magnesium (Mg; Osorio et al. 2015), Al (Nordlander \& Lind 2017), silicon (Si; Amarsi \& Asplund 2017), calcium (Ca; Mashonkina et al. 2008), titanium (Ti; Sitnova et al. 2020), Fe (Amarsi et al. 2016), and Ba (Mashonkina et al. 2008).

As we targeted stars in the bulge, which is over $5 \mathrm{kpc}$ away from the Sun, we expect most of our stars to be giants with $\log g<3$ dex given that only giants would be sufficiently luminous to be observed at the bulge. However, the results from COMBS II indicate that our target selection has been contaminated by a number of nearby disc stars. Therefore, we require a synthetic grid with a wide range of possible parameters, including dwarf, giant, metal-rich, and metal-poor stars. Our grid covers the following range:

(i) $2500 \mathrm{~K} \leq T_{\text {eff }} \leq 6500 \mathrm{~K}$, steps $=250 \mathrm{~K}$;

(ii) $-0.5 \mathrm{dex} \leq \log g \leq 5 \mathrm{dex}$, steps $=0.25 \mathrm{dex}$;

(iii) $-5 \mathrm{dex} \leq[\mathrm{M} / \mathrm{H}] \leq 0.75 \mathrm{dex}$, steps $=0.25 \mathrm{dex}$.

We scale the microturbulence $\left(v_{\text {micro }}\right)$ with $T_{\text {eff }}$ using the relationship calibrated from the Gaia-ESO survey (Smiljanic et al. 2014):

$$
\begin{aligned}
v_{\text {micro }}= & 1.1+1.0 \times 10^{-4} \times\left(T_{\text {eff }}-5500\right) \\
& +4.0 \times 10^{-7} \times\left(T_{\text {eff }}-5500\right)^{2}
\end{aligned} .
$$


We also scale the global $[\alpha / \mathrm{Fe}]$ with $[\mathrm{M} / \mathrm{H}]$ as follows:

$[\alpha / \mathrm{Fe}]= \begin{cases}0, & \text { if }[\mathrm{M} / \mathrm{H}] \geq 0 \\ -0.4 \times[\mathrm{M} / \mathrm{H}], & \text { if }-1 \leq[\mathrm{M} / \mathrm{H}] \leq 0 \\ 0.4, & \text { if }[\mathrm{M} / \mathrm{H}]<-1,\end{cases}$

in order to match the model atmospheres as well as empirical MW chemical evolution.

Following Carroll (1933a,b), we add a convolution term to account for rotational $(V \sin i)$ and instrumental broadening. We allow this term to vary between $0 \mathrm{~km} \mathrm{~s}^{-1} \leq V \sin i \leq 30 \mathrm{~km} \mathrm{~s}^{-1}$. However, since we have two unique parts of our spectra (HR06 and HR21) that have different wavelength resolutions $(R \approx 24300$ and 18000 , respectively) the convolution term must be different for each part. Therefore, we multiply the convolution term by 1.35 (the ratio of the resolutions) before applying it to the HR21 spectra. We attempt to fit the convolution terms for the HR21 and HR06 spectra separately, but the degeneracy between the effect of $\log g$ and convolution on the $\mathrm{CaT}$ is too strong. Therefore, we must use what we know about the convolution from the HR06 spectra to constrain the HR21 convolution. To interpolate between grid points, we use a piecewise linear interpolator.

In order to avoid getting stuck in a local minimum when performing the $\chi^{2}$ fit, we ensure that we start with an accurate guess for the stellar parameters. We do this by performing a quick crosscorrelation with a grid of model spectra that is similar, but smaller than our grid for the $\chi^{2}$ fit. This smaller grid covers the following range:

(i) $3500 \mathrm{~K} \leq T_{\text {eff }} \leq 6500 \mathrm{~K}$, steps $=250 \mathrm{~K}$;

(ii) $0.5 \mathrm{dex} \leq \log g \leq 4 \mathrm{dex}$, steps $=0.5 \mathrm{dex}$;

(iii) $-5 \mathrm{dex} \leq[\mathrm{M} / \mathrm{H}] \leq 0.5 \mathrm{dex}$, steps $=0.5 \mathrm{dex}$.

There are many observational and modelling effects that may cause our model spectra to differ from the observed spectra in ways that can negatively impact the fit. For example, the cores of strong lines, like the CaT, are known to be strongly impacted by NLTE, even when using departure coefficients for population levels. Therefore, we mask pixels that are not well matched by the model spectra in order to minimize their impact on the spectral fitting. To do this, we compare our model spectra to Gaia Benchmark stars (GBSs; BlancoCuaresma et al. 2014). As these stars are observed in the Gaia-ESO survey (Gilmore et al. 2012), they have GIRAFFE HR21 spectra. However, they do not have HR06 spectra. Instead, we download reduced HARPS spectra (Mayor et al. 2003) from the ESO archive ${ }^{3}$ and degrade the resolution and wavelength coverage to match that of HR06 spectra. We then compare the observed spectra to synthesized spectra of the corresponding parameters derived in Jofré et al. (2014) and Heiter et al. (2015). We mask any pixels that differ from the observed spectra by $>0.1$ in normalized flux. As the ability of the synthesis to accurately reproduce each pixel of the observed spectra is a function of the stellar parameters, we make the masks using four different benchmark stars depending on the stellar parameters. Specifically, we use the initial guess parameters to choose between four different spectra: (1) for metal-poor giants ( $\log g<2.5$ dex and $[\mathrm{M} / \mathrm{H}] \leq-1.5 \mathrm{dex})$ we use HD 122563, (2) for metal-rich giants $(\log g<2.5 \mathrm{dex}$ and $[\mathrm{M} / \mathrm{H}]>-1.5 \mathrm{dex})$ we use Arcturus, (3) for metal-poor sub-giants/dwarfs $(\log g \geq 2.5$ and $[\mathrm{M} / \mathrm{H}] \leq-1.5 \mathrm{dex})$ we use HD 140283, and (4) for metal-rich sub-giants/dwarfs (log $g$ $\geq 2.5 \mathrm{dex}$ and $[\mathrm{M} / \mathrm{H}]>-1.5 \mathrm{dex})$ we use $\epsilon$ For.

${ }^{3}$ https://archive.eso.org/scienceportal/
In addition to the masking, we also use the difference between the observed benchmark spectra and the corresponding model spectra as an uncertainty term in our fit $\left(\sigma_{\text {synth }}\right)$. Therefore, we essentially underweight pixels in the $\chi^{2}$ fit that are not well reproduced by the model spectra. We add this term in quadrature with the flux uncertainties. We then use this combined uncertainty in the $\chi^{2}$ fit.

Thus, the $\chi^{2}$ equation that we minimize is

$\chi^{2}=\sum \frac{(\text { observed }- \text { model })^{2}}{\left(\sigma_{\text {flux }}^{2}+\sigma_{\text {synth }}^{2}\right)}$,

where observed is the observed flux, model is the synthesis flux, $\sigma_{\text {flux }}$ is the flux uncertainties, and $\sigma_{\text {synth }}$ is the synthesis uncertainty as described above. We use the Nelder-Mead algorithm to find the global minimum.

Of the 387 stars for which we attempt stellar parameter analysis, we find a number of stars that we are unable to fit. Upon visual inspection, it is clear that one of these stars (899.0) is a CEMP-s star from the overwhelming $\mathrm{C}_{2}$ Swan band features and strong $\mathrm{Ba}$ line absorption at $4554 \AA$. However, we do not report results for this star in this work, as it requires separate analysis and will be thoroughly studied in a future instalment of the COMBS survey. We also find two stars (1386.0 and 1659.0) that may show $C$ enhancement and are unable to be fitted by our pipeline. Although we will attempt to analyse them in future work with 899.0, these stars are not obviously CEMP stars. In addition, we find seven stars that continually give solutions at the edge of our grid, with $T_{\text {eff }}=6500 \mathrm{~K}$. We exclude these stars given that solutions at the edge of the grid are not trustworthy.

Upon visual inspection, we choose to only perform elemental abundance analysis for spectra with SNR $>20 \mathrm{pixel}^{-1}$. Of the 377 stars with SNR $>10$ pixel $^{-1}$ for which we have stellar parameter solutions, 344 have SNR $>20$ pixel $^{-1}$. Furthermore, 319 of these stars have a match in Gaia DR2 within 1 arcsec and a Gaia DR2-renormalized unit weight error (ruwe) $<1.4$ (Lindegren 2018). Therefore, only these 319 stars have measured dynamics from COMBS II. For the rest of this work, we focus on these 319 stars since combining the dynamical analysis with the measured chemistry is essential to the goal of this work.

We present a Kiel diagram of these 319 stars in Fig. 1. The centre of the points is coloured by the SNR of the HR06 spectra. We also create rings around the points that are coloured by the metallicity. Along with our data, we also show 10 Gyr MIST isochrones with various metallicities (Paxton et al. 2011, 2013, 2015; Choi et al. 2016; Dotter 2016). Our data are well represented by these models, which is consistent with MW bulge age estimates (Zoccali et al. 2003).

\subsection{Stellar parameter uncertainties}

In order to accurately evaluate the uncertainties on the stellar parameters, we must take into account the internal uncertainties, caused by noise in the data and biases in the fitting procedure, as well as the external uncertainties, caused by imperfections in the model spectra. To account for the internal uncertainties, we aim to evaluate the precision of our fitting procedure as a function of SNR. To do this, we run our fitting procedure on synthetic spectra with known stellar parameters and various SNRs. To create these spectra, we use the same synthesis method as was used to create the model spectra grid and we randomly select 100 sets of parameters where $3500 \mathrm{~K} \leq T_{\text {eff }} \leq 5500 \mathrm{~K}, 0.5 \mathrm{dex} \leq \log g \leq 4 \mathrm{dex}$, and $-5 \mathrm{dex} \leq$ $[\mathrm{M} / \mathrm{H}] \leq 0.5$ dex. After synthesizing these 100 spectra with random parameters, we add synthetic Gaussian noise according to the desired SNR. As we aim to evaluate the precision of our method across the entire SNR range of our observed sample, we add noise in order 

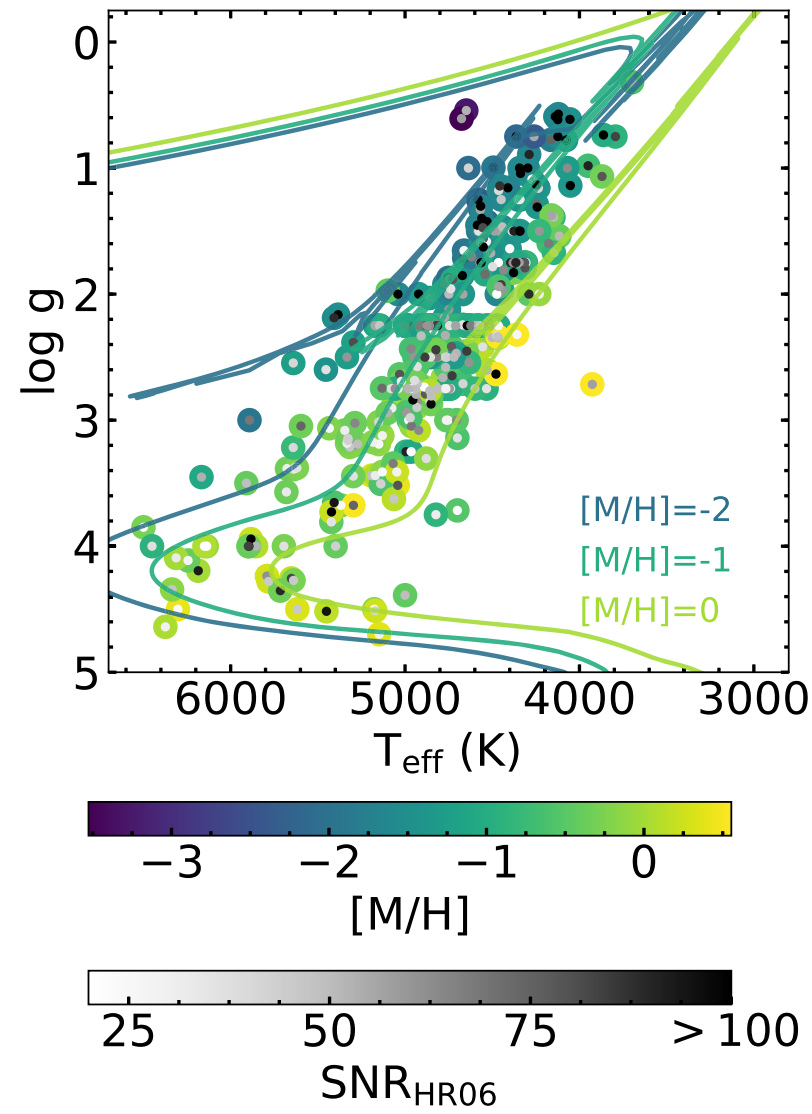

Figure 1. Kiel diagram for our sample of 319 stars for which we report stellar parameters and elemental abundances. The points are coloured by SNR in the centre and metallicity in the outer ring. We also plot $10 \mathrm{Gyr}$ MIST isochrones with $[\mathrm{M} / \mathrm{H}]=0,-1$, and $-2 \mathrm{dex}$ in green, light blue, and dark blue lines, respectively. These lines match the metallicity colour scale. Our data are well represented by the models, with the exception of outliers that typically have low SNR.

to create spectra with 10 pixel $^{-1} \leq \mathrm{SNR} \leq 250$ pixel $^{-1}$ in steps of 10 pixel $^{-1}$. We do this for each of our 100 synthetic spectra with random parameters, resulting in a total of 2500 spectra with varying parameters and SNRs with which we can evaluate our precision.

We put each of the 2500 synthetic spectra through our parameter analysis pipeline and compare the derived parameters to the true values. For every 100 spectra with the same SNR, we take the standard deviation of the differences between the derived and true values. We use this value as our estimate for the internal precision at that SNR. Therefore, we have internal precision estimates for 25 different SNR values.

We show the calculated internal precision for a range of SNRs in Fig. 2. We fit exponentially decreasing functions to estimate the precision, or internal uncertainty, as a function of SNR. We find that the internal uncertainties are best described as

$\sigma_{T_{\text {eff }} \text {,int }}=345 \mathrm{~K} \mathrm{e}^{-0.082 \times \mathrm{SNR}}+36 \mathrm{~K}$

$\sigma_{\log g, \text { int }}=0.653 \mathrm{e}^{-0.086 \times \mathrm{SNR}}+0.123$

$\sigma_{[\mathrm{M} / \mathrm{H}], \mathrm{int}}=0.173 \mathrm{e}^{-0.049 \times \mathrm{SNR}}+0.051$.

Therefore, we can use these equations to evaluate the $T_{\text {eff }}, \log g$, and $[\mathrm{M} / \mathrm{H}]$ internal uncertainties for each of our stars. Specifically, we calculate the internal uncertainties using the SNR estimates for the HR06 spectra that are always lower than the SNR estimates for the

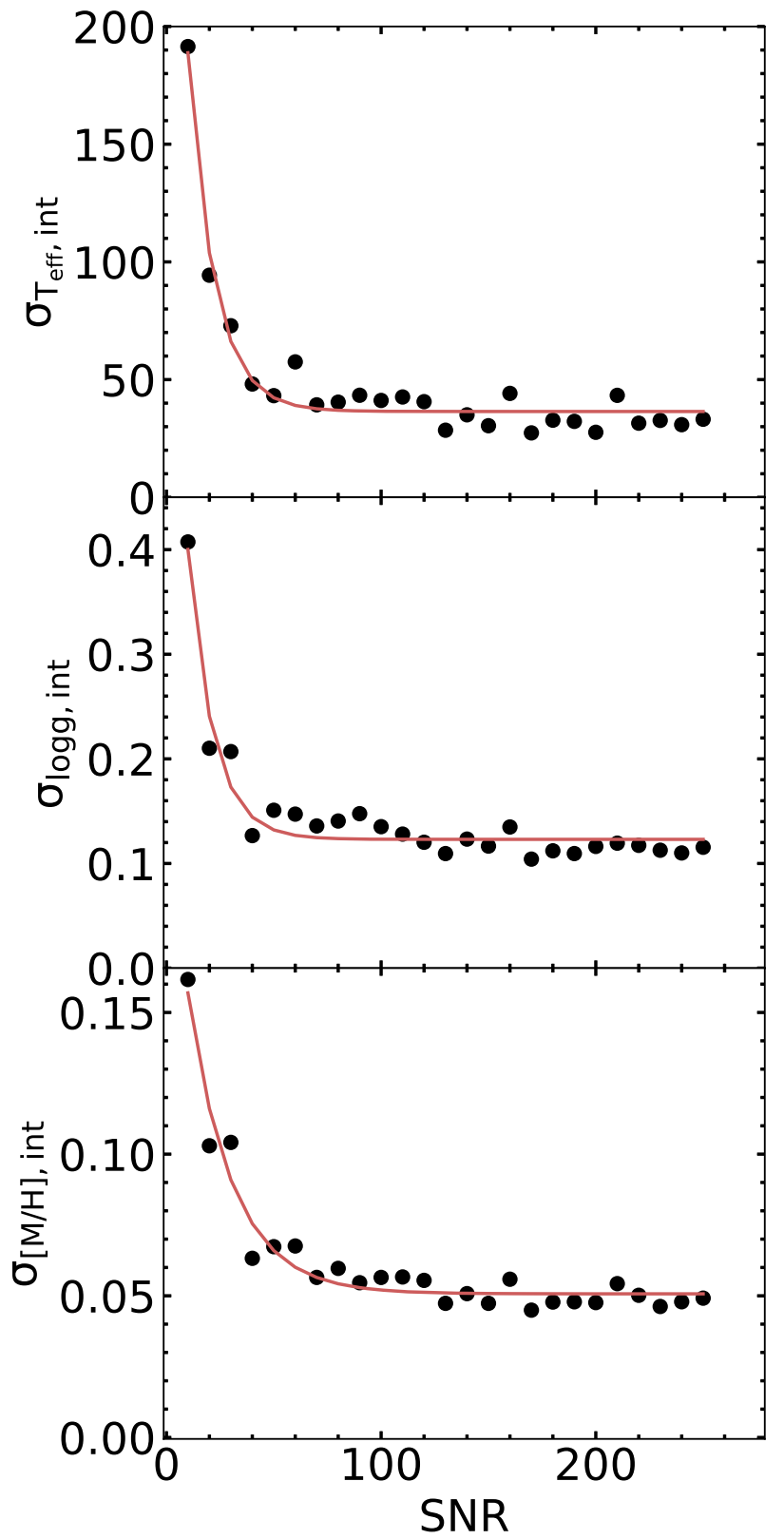

Figure 2. The estimates of the internal uncertainty for $T_{\text {eff }}, \log g$, and $[\mathrm{M} / \mathrm{H}]$ as a function of SNR. The black points represent the standard deviations of the differences between the derived and synthesized parameters for 100 random synthetic spectra as a function of the SNR. The red lines are the bestfitting exponentially decreasing functions that are then used to determine the internal uncertainty estimates for our observed data.

HR21 spectra. Given that the SNR was the same for both HR06 and HR21 in our synthetic analysis, we may be slightly overestimating our uncertainties since the HR21 spectra will have higher SNR in our observations.

To evaluate the external uncertainties, we use a sample of 10 Gaia Benchmark giant and subgiant stars. These stars are common calibration stars that are frequently used to evaluate the accuracy and precision of stellar parameter pipelines (e.g. Smiljanic et al. 2014; Buder et al. 2018; Duong et al. 2019a). They are especially useful to compare to spectroscopically derived parameters since their reference $T_{\text {eff }}$ and $\log g$ values are determined independently from their spectra. Specifically, the bolometric flux and angular diameter 

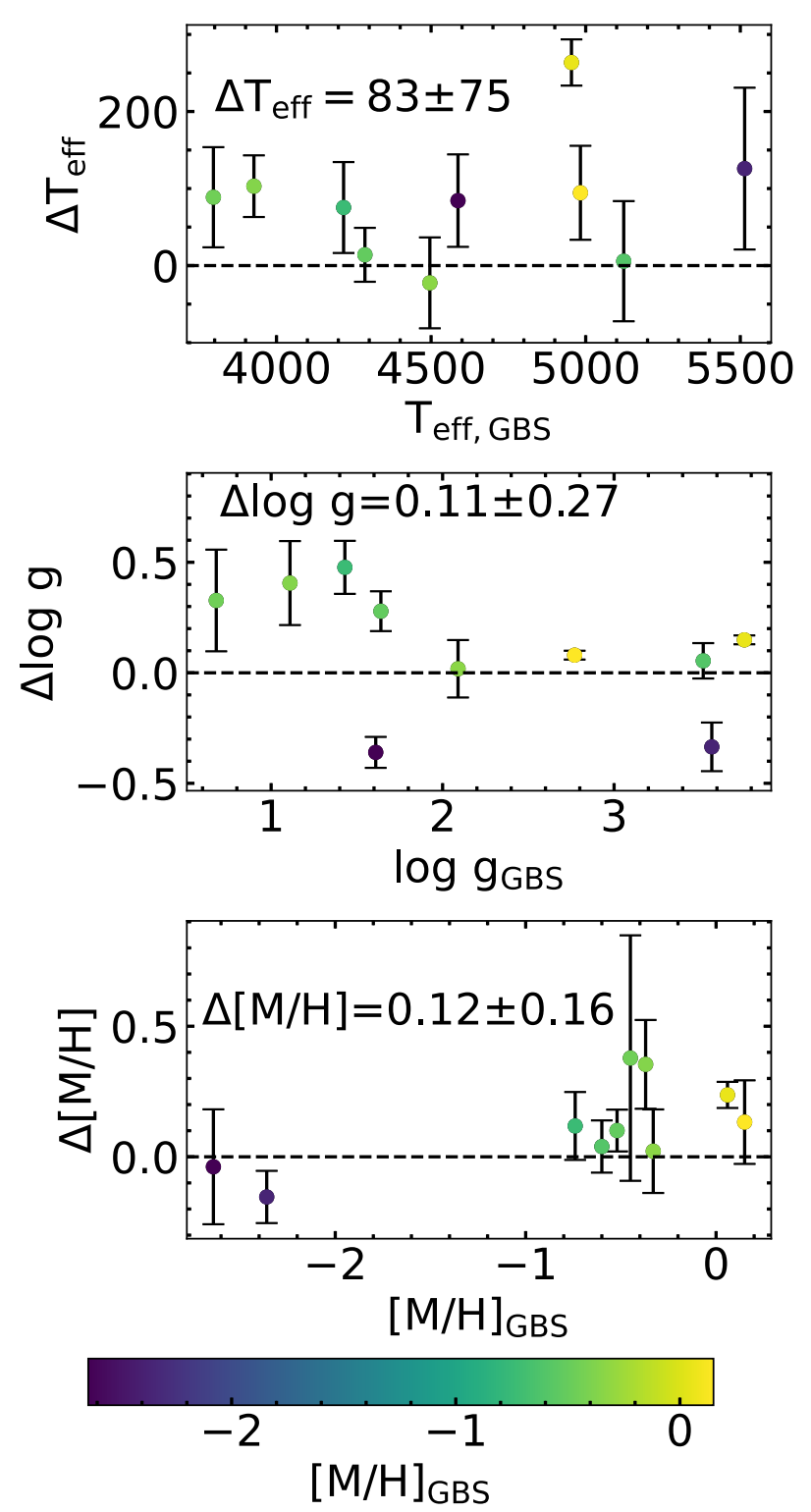

Figure 3. The differences between our derived values and the reference values from Heiter et al. (2015) and Jofré et al. (2014) for 10 GBSs. The differences are (this work - GBS). The points are coloured by the reference metallicity. The error bars shown are for the reference values. We also provided the mean and standard deviation of the differences for each parameter in the black text. The standard deviation of the differences is used as our external uncertainty estimate for the given parameter.

are used to determine $T_{\text {eff }}$. The value of $\log g$ is then determined using the angular diameter and mass estimate.

In Fig. 3, we show the comparison of our results to the reference values for $10 \mathrm{GBSs}$. We colour each point by metallicity in order to track the impact of metallicity on the $T_{\text {eff }}$ and $\log g$ determination. The differences on the $y$-axis are (this work-GBS). For $T_{\text {eff }}$, we find a mean bias of $83 \mathrm{~K}$ with a standard deviation of $75 \mathrm{~K}$. For $\log g$, we find a bias of 0.11 dex with a standard deviation of 0.27 dex. Lastly, for $[\mathrm{M} / \mathrm{H}]$, we find a bias of 0.12 dex with a standard deviation of 0.16 dex. However, it is important to note that we are comparing our global metallicity value to their metallicity derived from only Fe lines, which may introduce some bias as a function of $[\mathrm{M} / \mathrm{H}]$. Overall, these results are comparable to the HERBS survey that has a similar sample and analysis method as this work (see fig. A1 in Duong et al. 2019a).

We use the derived standard deviations of the differences for $T_{\text {eff }}$, $\log g$, and $[\mathrm{M} / \mathrm{H}]$ as our external uncertainty estimates. Our overall uncertainty estimate is calculated by adding the internal and external uncertainty estimates in quadrature. The external uncertainty is larger than the internal uncertainty for $T_{\text {eff }}, \log g$, and $[\mathrm{M} / \mathrm{H}]$ at high SNR $\left(\mathrm{SNR} \gtrsim 100 \mathrm{pixel}^{-1}\right)$. Therefore, the external uncertainty dominates our stellar parameter uncertainties for stars with SNR $\gtrsim 100 \mathrm{pixel}^{-1}$ and the internal uncertainty only becomes important at SNR $\lesssim 50$ pixel $^{-1}$.

\section{ELEMENTAL ABUNDANCE ANALYSIS}

Once the stellar parameters are determined, we perform a line-byline $\chi^{2}$ fit to determine the individual elemental abundances. For each line, we compute synthetic spectra using the same method as in the stellar parameter analysis, including all of the same NLTE departure coefficient grids. Specifically, we compute five different spectra with $[\mathrm{X} / \mathrm{Fe}]=(-0.6,-0.3,0.0,0.3,0.6)$ dex. If the derived solution is $[\mathrm{X} / \mathrm{Fe}]=0.6$ or -0.6 dex, we repeat the analysis but add or subtract 1 dex from the synthesized $[\mathrm{X} / \mathrm{H}]$ values. We use the derivatives of the spectrum with respect to the elemental abundance to determine the pixel selection. Explicitly, going out from the line core, we include all pixels until the derivative changes sign or becomes $<0.01 \mathrm{dex}^{-1}$. However, we also force the minimum line window to be $0.2 \AA$ wide and the maximum line window to be $10 \AA$ wide. This method is similar to what is applied in other spectroscopic codes (e.g. the BACCHUS code; Hawkins et al. 2015; Masseron, Merle \& Hawkins 2016).

As the strength of absorption features is strongly dependent on the metallicity, we find that it is necessary to use a metallicitydependent line selection to avoid weak, blended, or saturated lines across our entire metallicity range. Specifically, we have a very metal poor $([\mathrm{M} / \mathrm{H}] \leq-2.0 \mathrm{dex})$, metal-poor $(-2.0 \mathrm{dex}<[\mathrm{M} / \mathrm{H}] \leq-0.5$ $\mathrm{dex})$, and metal-rich $([\mathrm{M} / \mathrm{H}]>-0.5 \mathrm{dex})$ line selection. However, we include many of the same lines between the selections to ensure continuity.

Although we report the abundance derived from each individual line, we use the mean of the lines as our final $[\mathrm{X} / \mathrm{H}]$ value. We report elemental abundances for $\mathrm{C}, \mathrm{Na}, \mathrm{Mg}, \mathrm{Al}, \mathrm{Si}, \mathrm{Ca}$, Ti, chromium $(\mathrm{Cr})$, $\mathrm{Mn}, \mathrm{Fe}$, zinc ( $\mathrm{Zn}$ ), Ba, and cerium (Ce). Of those, the only elements for which we do not use NLTE departure coefficient grids are C, $\mathrm{Cr}, \mathrm{Mn}, \mathrm{Zn}$, and Ce. We note that the NLTE effects of $\mathrm{Cr}$ and $\mathrm{Mn}$ are important when we constrain the enrichment source from the abundance pattern, in particular for low- $\alpha$ stars (Kobayashi et al. 2014).

For each atomic line, we determine an associated uncertainty for the derived abundance based on the $\chi^{2}$ fit. The uncertainty is the distance in abundance space from the minimum $\chi^{2}$ to where the reduced $\chi^{2}$ equals the minimum $\chi^{2}$ plus one (e.g. the FERRE ${ }^{4}$ code; Allende Prieto 2004; Allende Prieto et al. 2006, 2008; Allende Prieto, Hubeny \& Smith 2009). After visual inspection of 50 stars with varying SNR, we find that an individual line abundance uncertainty $\gtrsim 0.25$ dex tends to indicate an untrustworthy fit and requires further visual inspection to determine if the line fit should be discarded. We also inspect stars whose line-by-line scatter in the abundance is $\gtrsim 0.25 \mathrm{dex}$. For our final abundance uncertainties, we propagate the individual line-by-line abundance uncertainties through the mean.

\footnotetext{
${ }^{4}$ Available at http://hebe.as.utexas.edu/ferre.
} 
The result is the individual line-by-line uncertainties added in quadrature and then divided by the number of lines used.

\section{COMPARISON WITH ARGOS AND HERBS SURVEYS}

In order to test the accuracy and precision of our stellar parameters, we compare them to other large Galactic bulge surveys. Specifically, we compare to the ARGOS survey that uses $R \sim 11000$ spectra of $\sim 28000$ stars (Freeman et al. 2013). This survey measured the RV, $T_{\text {eff }}, \log g,[\mathrm{Fe} / \mathrm{H}]$, and $[\alpha / \mathrm{Fe}]$ ratio of their programme stars. Our work has 26 stars in common with the ARGOS survey. In addition, we also compare to the HERBS survey that uses $R \sim 28000$ spectra of 832 stars (Duong et al. 2019a,b). However, we only observed three stars in common with the HERBS survey, which is not enough for a thorough comparison. Fortunately, the HERBS survey performs a detailed comparison with the ARGOS survey. Therefore, we can compare to the HERBS survey through a comparison with the ARGOS survey.

In Fig. 4, we show the comparison between our derived stellar parameters and the values from the ARGOS survey. The differences shown are (this work - ARGOS). The points are coloured by the ARGOS-derived metallicity. The error bars are the uncertainties on our derived parameters. In the bottom panel, we compare the ARGOS metallicity to our $[\mathrm{Fe} / \mathrm{H}]$ value derived from $\mathrm{Fe}$ lines, rather than the global $[\mathrm{M} / \mathrm{H}]$ derived during the stellar parameter analysis. However, we have also performed the comparison using the global $[\mathrm{M} / \mathrm{H}]$ and found the results to be similar to $[\mathrm{Fe} / \mathrm{H}]$. We find that the mean difference in $T_{\text {eff }}$ is $-77 \mathrm{~K}$ with a standard deviation of $248 \mathrm{~K}$. The mean difference in $\log g$ is 0.39 dex with a standard deviation of 0.58 dex, while the mean difference in $[\mathrm{Fe} / \mathrm{H}]$ is 0.14 dex with a standard deviation of 0.26 dex.

When comparing to the ARGOS survey, the HERBS survey reports the median, $1 \sigma$, and standard deviation (after excluding $3 \sigma$ outliers) of the differences between derived stellar parameters (Duong et al. 2019a). They find a median difference in $T_{\text {eff }}$ of $-64 \mathrm{~K}$, which is consistent with our value of $-77 \mathrm{~K}$. However, our $1 \sigma$ value ( $246 \mathrm{~K})$, which is also very similar to our standard deviation before $(248 \mathrm{~K})$ and after excluding $3 \sigma$ outliers $(248 \mathrm{~K})$, is significantly larger than the value reported by the HERBS survey $(117 \mathrm{~K})$. We expect that this difference is largely due to the different metallicity distribution of our sample. As the ARGOS survey derives $T_{\text {eff }}$ using the photometric colours, it is reasonable to assume that their $T_{\text {eff }}$ precision would be metallicity dependent, given that metallicity also impacts the photometric colours. Specifically, it is possible that the ARGOS survey may have worse $T_{\text {eff }}$ precision for metalpoor stars. In fact, Freeman et al. (2013) notes that using different empirical $T_{\text {eff }}$ - colour calibrations lead to differences in $T_{\text {eff }}$ estimates up to $200 \mathrm{~K}$ for metal-poor stars (Bessell, Castelli \& Plez 1998; Alonso, Arribas \& Martínez-Roger 1999). Given that our survey is significantly more metal poor than the HERBS survey, we would therefore expect the ARGOS precision to be worse for our sample than the HERBS sample. Furthermore, we note that for the three stars we have in common with the HERBS survey we find that the standard deviation for the differences in $T_{\text {eff }}$ between our values and the HERBS values is $168 \mathrm{~K}$. In addition, it is interesting to note that when comparing APOGEE DR16 stellar parameters (Ahumada et al. 2020) to ARGOS, Wylie et al. (2021) find that the differences in $T_{\text {eff }}$ have a standard deviation of $321 \mathrm{~K}$, which is significantly larger than our value of $248 \mathrm{~K}$.

For $\log g$, we find that our results are very consistent with the HERBS survey. Specifically, our median difference is 0.39 dex while
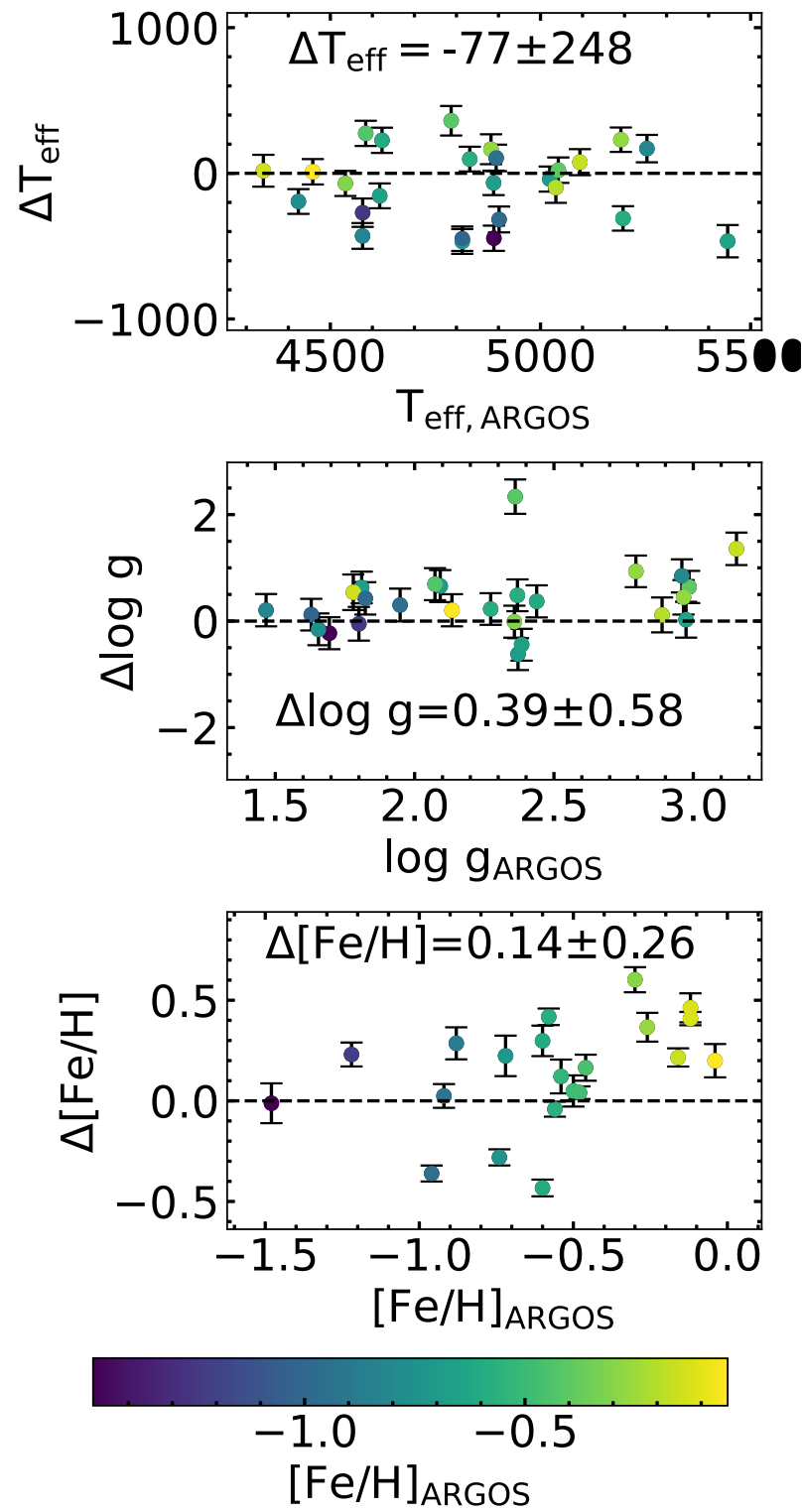

Figure 4. Comparison of the derived stellar parameters compared to results from the ARGOS survey (Freeman et al. 2013) for 26 stars in common. The differences shown are (this work - ARGOS). The points are coloured by the $[\mathrm{Fe} / \mathrm{H}]$ from the ARGOS survey to ensure there are no trends in accuracy and precision of the stellar parameters with $[\mathrm{Fe} / \mathrm{H}]$. The error bars are the uncertainties for our derived parameters. The text gives the mean and standard deviations of the differences for each stellar parameter.

the HERBS survey reports a median difference of 0.29 dex. The $1 \sigma$ difference for our work is 0.30 dex while the HERBS survey finds a $1 \sigma$ of 0.29 dex. Last, the standard deviation we find after removing $3 \sigma$ outliers is 0.34 dex, while the HERBS survey reports 0.38 dex. These results indicate that our stellar parameter analysis is consistent with the results from the HERBS survey.

Last, for $[\mathrm{Fe} / \mathrm{H}]$, we find a median difference of 0.20 dex between our $[\mathrm{Fe} / \mathrm{H}]$ and the values from ARGOS, while the HERBS survey reports a value of 0.04 dex. From Fig. 4 , it is clear that our large bias is mostly due to our $[\mathrm{Fe} / \mathrm{H}]$ being significantly larger than the ARGOS values for stars with $[\mathrm{Fe} / \mathrm{H}]>-0.5$ dex in ARGOS. We note that the median offset between our $[\mathrm{Fe} / \mathrm{H}]$ results and the HERBS survey for the three stars in common is 0.03 dex. It is also important to note that 


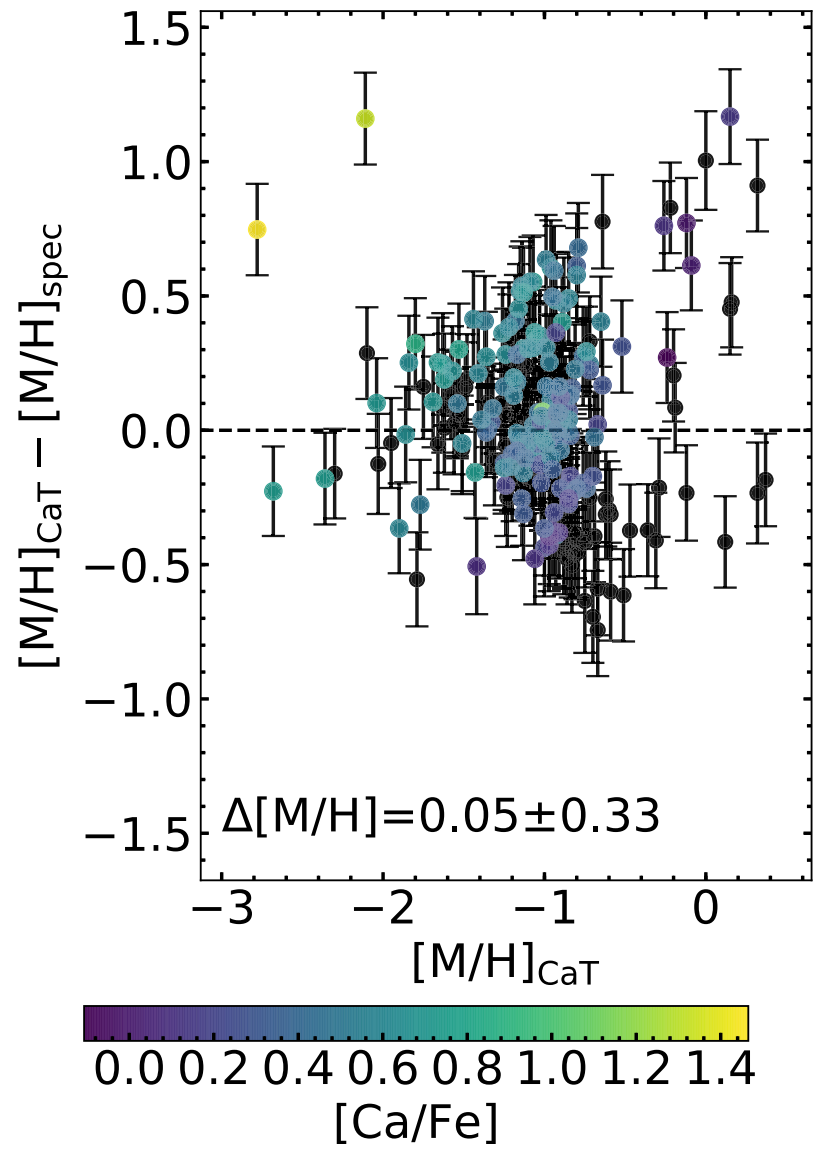

Figure 5. Comparison between metallicity estimates from the CaT presented in COMBS II to the $[\mathrm{M} / \mathrm{H}]$ results presented in this work. The points are coloured by the $[\mathrm{Ca} / \mathrm{Fe}]$ abundance when available. The error bars shown are the uncertainty estimates on $[\mathrm{M} / \mathrm{H}]$ from this work. The black text shows the bias, or mean difference, $(0.05 \mathrm{dex})$ and the standard deviation of the differences $(0.33 \mathrm{dex})$.

these three stars have -0.7 dex $<[\mathrm{Fe} / \mathrm{H}]_{\text {HERBS }}<-0.3$ dex, which is the same range where we are most inconsistent with ARGOS. We find that our spread in $[\mathrm{Fe} / \mathrm{H}]$ differences with ARGOS is similar to the differences reported in the HERBS survey. Specifically, we find a $1 \sigma$ of 0.17 dex while HERBS reports a $1 \sigma$ of 0.14 dex. After removing $3 \sigma$ outliers, we find a standard deviation of $0.17 \mathrm{dex}$. While using the same method, HERBS finds a standard deviation of 0.16 dex. Therefore, we find our stellar parameter results to be generally consistent with the HERBS survey.

\section{METALLICITY DISTRIBUTION FUNCTION}

The MDF of the Galactic bulge is well studied through photometric and spectroscopic surveys and is primarily composed of a metalrich population with $[\mathrm{Fe} / \mathrm{H}]>-1 \mathrm{dex}$ (Zoccali et al. 2008, 2017; Bensby et al. 2013, 2017; Johnson et al. 2013; Ness et al. 2013a; Rojas-Arriagada et al. 2014, 2017, 2020; García Pérez et al. 2018; Duong et al. 2019a; Johnson et al. 2020). In this work, we have used SkyMapper photometry to target the metal-poor tail of the Galactic bulge MDF. Therefore, we expect our sample to have an MDF that is on the metal-poor end with $[\mathrm{Fe} / \mathrm{H}]<-1$ dex.

In COMBS II, metallicity estimates were determined from the CaT using the same spectra presented in this work. In Fig. 5, we show a comparison between the results presented in COMBS II and

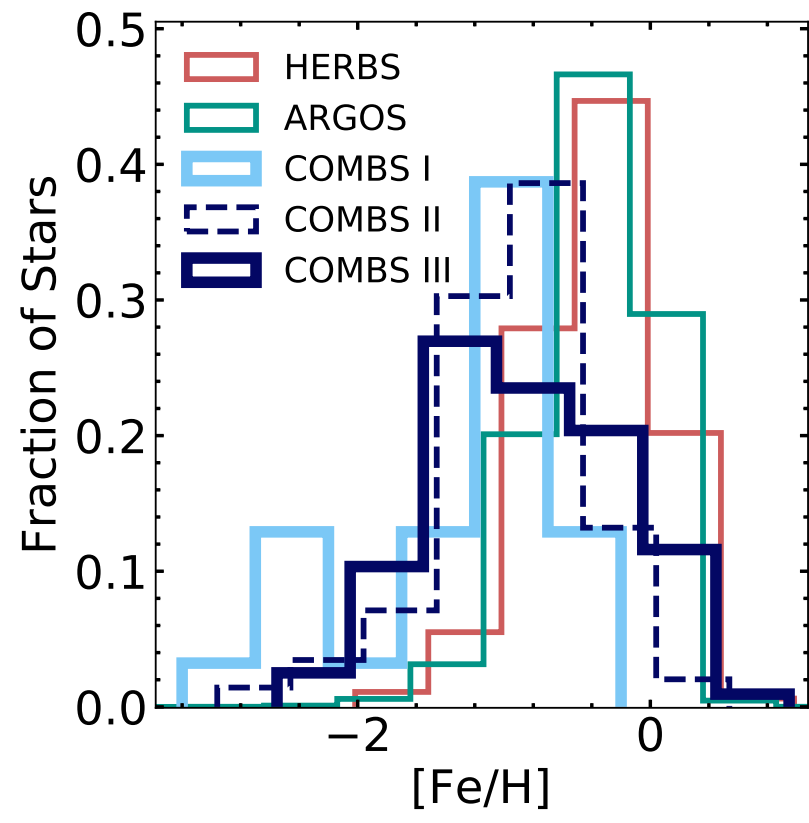

Figure 6. The MDF for our results (dark blue solid line), compared to results from COMBS II (dark blue dashed line), COMBS I (light blue solid line), ARGOS (green solid line; Freeman et al. 2013; Ness et al. 2013a), and HERBS (red solid line; Duong et al. 2019a). Our MDF is more metal poor than surveys that did not target metal-poor stars (ARGOS and Bensby et al. 2017). Therefore, our selection of metal-poor stars with SkyMapper photometry was successful.

the $[\mathrm{M} / \mathrm{H}]$ results determined in the stellar parameter analysis of this work. For this figure, we only show results for stars with $\log g \leq 3$ dex as our CaT method was designed to be applied to giant stars similar to previous work on metallicity estimates from the CaT (Armandroff \& Zinn 1988; Armandroff \& Da Costa 1991; Olszewski et al. 1991; Cole et al. 2004; Battaglia et al. 2008; Starkenburg et al. 2010; Li et al. 2017). The error bars shown are those derived for the $[\mathrm{M} / \mathrm{H}]$ value in this work. We colour the points by the $[\mathrm{Ca} / \mathrm{Fe}]$ abundance. The metallicity estimates from COMBS II are generally consistent with the $[\mathrm{M} / \mathrm{H}]$ results from this work, with only a 0.05 dex bias. The standard deviation of the differences is 0.33 dex that is only slightly larger than the uncertainty on the metallicity estimates from the CaT (0.22 dex) added in quadrature with the mean $[\mathrm{M} / \mathrm{H}]$ uncertainty in this work $(0.17$ dex $)$.

We present the MDF of our sample in Fig. 6 using the derived $[\mathrm{Fe} / \mathrm{H}]$ abundances (dark blue solid line). We also show the results from COMBS II (dark blue dashed line), COMBS I (light blue solid line), the ARGOS survey (green solid line; Freeman et al. 2013; Ness et al. 2013a), and the HERBS survey (red solid line; Duong et al. 2019a). Our MDF peaks at $[\mathrm{Fe} / \mathrm{H}] \approx-1 \mathrm{dex}$, while the results for the surveys that did not target metal-poor stars (ARGOS and HERBS) peak at $[\mathrm{Fe} / \mathrm{H}] \gtrsim-0.5$ dex. Therefore, our use of SkyMapper photometry to select metal-poor stars was successful. However, we have relatively fewer stars with $[\mathrm{Fe} / \mathrm{H}]<-2$ dex compared to COMBS I. This is expected, given that the most promising metalpoor targets were prioritized for the high-resolution UVES spectra that were presented in COMBS I. Compared to COMBS II, we see a stronger metal-rich tail that broadens the MDF. This was likely missed in COMBS II because the $[\mathrm{Ca} / \mathrm{Fe}]$ ratio decreases at $[\mathrm{Fe} / \mathrm{H}]$ $>-1$ dex. This causes a smaller increase in $[\mathrm{Ca} / \mathrm{H}]$ for a given increase in $[\mathrm{Fe} / \mathrm{H}]$. Therefore, $[\mathrm{Fe} / \mathrm{H}]$ values estimated from $\mathrm{Ca}$ lines would be underestimated in this $[\mathrm{Fe} / \mathrm{H}]$ range. 


\section{ELEMENTAL ABUNDANCE RESULTS}

The chemical abundances of stars provide unique insight into the formation and evolution of stellar populations. However, in order to interpret the abundances, we need to contextualize our results in terms of other stellar populations and nucleosynthetic pathways. In this section, we present our abundance results and discuss the formation mechanisms for each element. We also compare our results with other MW populations and literature samples.

\subsection{C}

$\mathrm{C}$ is primarily produced in massive stars $\left(>10 \mathrm{M}_{\odot}\right)$ and low-mass asymptotic giant branch (AGB) stars. The $[\mathrm{C} / \mathrm{Fe}]$ yield is especially increased in low-mass stars where the Fe yield is essentially zero (Kobayashi, Karakas \& Umeda 2011a). In addition, high level of Cenhancement $([\mathrm{C} / \mathrm{Fe}]>1 \mathrm{dex})$ among metal-poor stars is thought to come from Population III supernovae, specifically faint supernovae (e.g. Nomoto, Kobayashi \& Tominaga 2013).

In this work, we measure elemental $\mathrm{C}$ abundances from the atomic line at $8727 \AA$. However, in stars with $[\mathrm{Fe} / \mathrm{H}]<-2$ dex, we find that this line is too weak to measure an accurate abundance from. Furthermore, as stars move up the red giant branch, they experience the second dredge-up that depletes the photospheric $\mathrm{C}$ abundance. To account for this depletion, we apply a correction factor to our derived $\mathrm{C}$ abundances. These correction factors come from Placco et al. (2014) and are a function of the $\log g,[\mathrm{Fe} / \mathrm{H}]$, and uncorrected $[\mathrm{C} / \mathrm{Fe}]$ ratio. We show the corrected abundances in Fig. 7 . We note that the shown literature abundances from Roederer et al. (2014) and Howes et al. (2016) have not been corrected.

At $[\mathrm{Fe} / \mathrm{H}] \gtrsim-1 \mathrm{dex}$, the $[\mathrm{C} / \mathrm{Fe}]$ ratio decreases with increasing $[\mathrm{Fe} / \mathrm{H}]$. This is consistent with chemical evolution models and the onset of Type Ia Supernovae (SNe Ia) that overproduce Fe with respect to $\mathrm{C}$. At $[\mathrm{Fe} / \mathrm{H}] \lesssim-1$ dex, generally $[\mathrm{C} / \mathrm{Fe}]>0$ dex. In order to reach this level of $\mathrm{C}$ enhancement, it is likely that inhomogeneous mixing needs to be taken into account, which allows AGB stars to contribute $\mathrm{C}$ yields at $[\mathrm{Fe} / \mathrm{H}] \lesssim-1.5$ dex (Kobayashi et al. 2014; Vincenzo \& Kobayashi 2018).

\section{$7.2 \alpha$-elements}

The $\alpha$-elements are generally divided into two categories based on their formation site. Specifically, the hydrostatic $\alpha$-elements $(\mathrm{Mg})$ primarily form in the hydrostatic burning phase of massive stars, while the explosive $\alpha$-elements ( $\mathrm{Ca}$ and $\mathrm{Si}$ ) are primarily produced through explosive nucleosynthesis of core-collapse, or Type II, supernovae (SNe II; Woosley \& Weaver 1995; Woosley, Heger \& Weaver 2002). Specifically, $\mathrm{Mg}$ is produced from $\mathrm{C}$ and neon $(\mathrm{Ne})$ burning, while $\mathrm{Si}$ and $\mathrm{Ca}$ are primarily synthesized from explosive $\mathrm{O}$ burning. Thus, the yields of explosive $\alpha$-elements depend on the explosion energy (Kobayashi et al. 2006). Although they have different formation sites, the hydrostatic and explosive elements tend to trace each other as they are usually mixed during supernova explosions and dispersed into the interstellar medium (ISM). At low metallicities, before the onset of SNe Ia, the $\alpha$-element abundances are generally indicative of the IMF of the enriching stellar population, given that their yields in SNe II are mass dependent. On the other hand, $\mathrm{SNe}$ Ia overproduce Fe with respect to the $\alpha$-elements and cause the $[\alpha / \mathrm{Fe}]$ ratio to decrease. Therefore, the $[\mathrm{Fe} / \mathrm{H}]$ value at which the $[\alpha / \mathrm{Fe}]$ ratio begins to decrease specifies the amount of Fe built up by SNe II before the onset of SNe Ia. Furthermore, the behaviour of the $[\alpha / \mathrm{Fe}]$ ratio as a function of $[\mathrm{Fe} / \mathrm{H}]$ is indicative of the star formation time-scale, where a short star formation time-scale leads to a large build-up of Fe in the ISM before the onset of SNe Ia.

\subsection{1 $\mathrm{Mg}$}

Mg abundances at $[\mathrm{Fe} / \mathrm{H}] \gtrsim-1$ dex are slightly higher in the bulge than in the disc (McWilliam \& Rich 1994; Rich \& McWilliam 2000; McWilliam \& Rich 2004; Fulbright et al. 2007; Johnson et al. 2014; Gonzalez et al. 2015; Bensby et al. 2017; Duong et al. 2019a). This is consistent with a shorter star formation time-scale causing a larger build-up of $\mathrm{Fe}$ and $\mathrm{Mg}$ from $\mathrm{SNe}$ II before the contribution from SNe Ia begins. As shown in Fig. 7, our results are consistent with the literature at $[\mathrm{Fe} / \mathrm{H}] \geq-1 \mathrm{dex}$.

At $[\mathrm{Fe} / \mathrm{H}] \lesssim-1$ dex, our abundance measurements generally continue to show high levels of $\mathrm{Mg}$ enhancement. Specifically, when compared to the EMBLA survey (Howes et al. 2015), our Mg abundances are generally higher. Furthermore, the $\mathrm{Mg}$ abundances reported by the EMBLA survey appear to be more consistent with a Galactic halo population, while our $\mathrm{Mg}$ abundances are higher than results from the halo (Yong et al. 2013; Roederer et al. 2014). However, it is difficult to draw strong conclusions here because there may be systematic offsets between surveys that impact the comparative results.

\subsection{2 $\mathrm{Ca}$ and $\mathrm{Si}$}

Measurements of $\mathrm{Ca}$ and $\mathrm{Si}$ abundances in the bulge at $[\mathrm{Fe} / \mathrm{H}] \gtrsim-1$ dex are generally higher than what is found in the disc (McWilliam \& Rich 1994; Rich \& McWilliam 2000; McWilliam \& Rich 2004; Fulbright et al. 2007; Johnson et al. 2014; Bensby et al. 2017; Duong et al. 2019a). However, our abundances at $[\mathrm{Fe} / \mathrm{H}] \gtrsim-1$ dex are slightly lower than literature values for the bulge. This is likely a systematic effect possible from our photometric targeting method, or offsets between surveys resulting from differences in analysis methods.

We measure high levels of $\mathrm{Ca}$ and $\mathrm{Si}$ enhancement at $[\mathrm{Fe} / \mathrm{H}] \lesssim$ -1 dex. Similar to $\mathrm{Mg}$, we find that our $\mathrm{Ca}$ and $\mathrm{Si}$ abundances are generally higher than what has been observed in the bulge by the EMBLA survey (Howes et al. 2015) and in the halo (Yong et al. 2013; Roederer et al. 2014). Our Ca abundances are especially high. This is interesting given that many Population III stars are thought to explode as PISNe that are theorized to have high Ca yields with $[\mathrm{Ca} / \mathrm{Fe}]$ as high as 2 dex. However, high $[\mathrm{Ca} / \mathrm{Fe}]$ itself does not suggest PISNe since faint $\mathrm{SNe}$ give high $[(\mathrm{Mg}, \mathrm{Si}, \mathrm{Ca}) / \mathrm{Fe}]$ as well. We discuss further signatures of PISNe, including the discriminatory $[\mathrm{Ca} / \mathrm{Mg}]$ ratio, in Section 9.

\subsection{Odd-Z elements}

The odd-Z elements are light elements that have an odd atomic number and therefore could not be produced by successive addition of $\alpha$ particles. In this work, we measure $\mathrm{Na}$ and $\mathrm{Al}$. The yields of $\mathrm{Na}$ and $\mathrm{Al}$ from $\mathrm{SNe}$ II are metallicity dependent, with higher yields from more metal-rich stars (Kobayashi et al. 2006). This leads to an increase in the $[(\mathrm{Na}, \mathrm{Al}) / \mathrm{Fe}]$ ratio with increasing $[\mathrm{Fe} / \mathrm{H}]$. However, $\mathrm{Fe}$ is overproduced relative to $\mathrm{Na}$ and $\mathrm{Al}$ in $\mathrm{SNe}$ Ia that causes the $[(\mathrm{Na}, \mathrm{Al}) / \mathrm{Fe}]$ ratio to decrease as these types of explosions become relevant.

\subsubsection{Na}

At $[\mathrm{Fe} / \mathrm{H}] \gtrsim-1$ dex, the bulge and disc show similar trends in [Na/Fe] (Bensby et al. 2017; Duong et al. 2019b). Consistent with our 

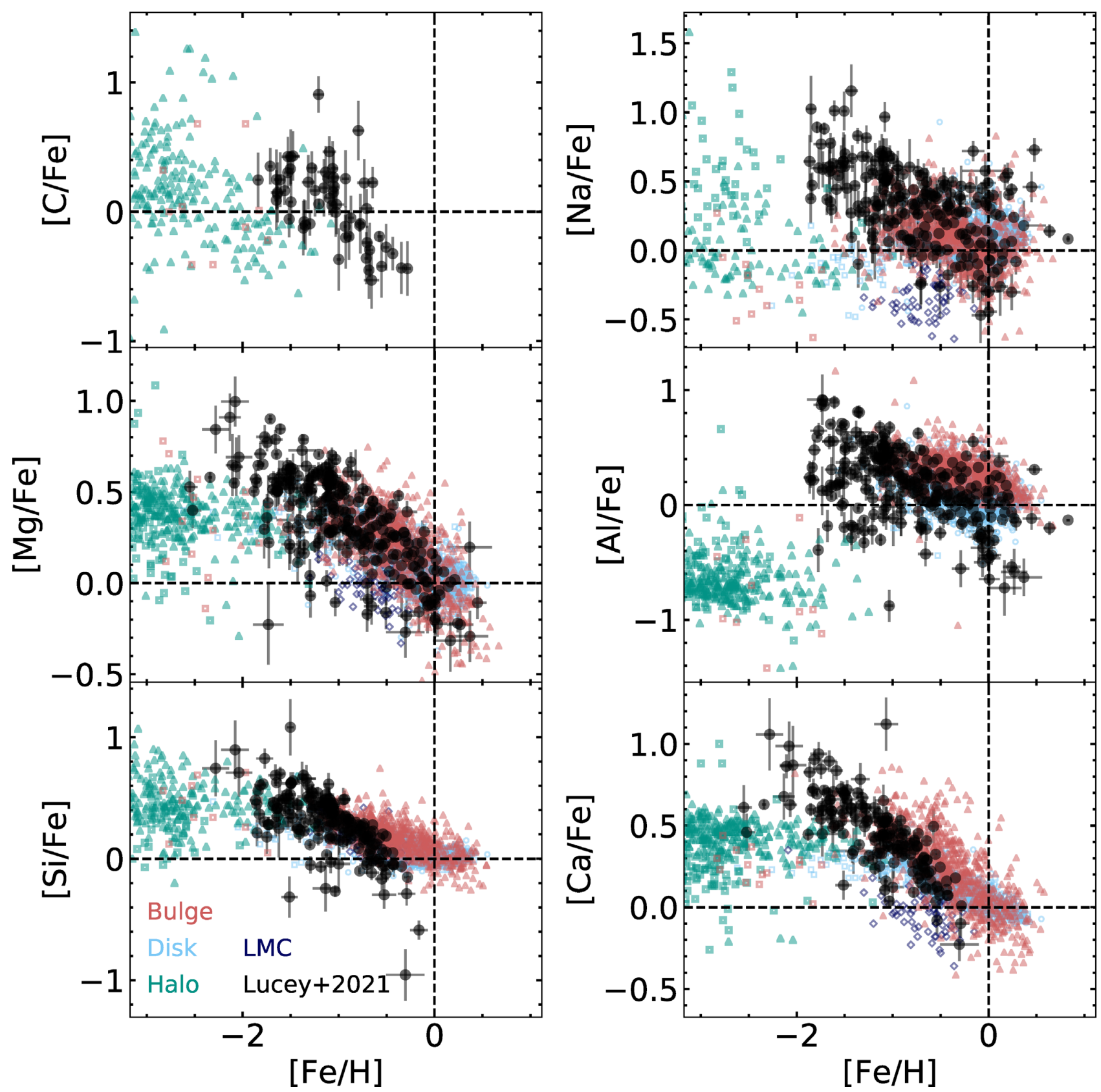

Figure 7. Light and $\alpha$-element abundances for all of the stars in our sample (black circles) compared to other MW samples from the literature. Specifically, we show other MW bulge samples in red, including results from the HERBS survey (red open triangles; Duong et al. 2019a,b) and results from the EMBLA survey (red open squares; Howes et al. 2016). Also shown are abundances for the halo (green), the Large Magellanic Cloud (LMC; dark blue), and the disc (light blue). The halo abundances are from Roederer et al. (2014, green triangles) and Yong et al. (2013, green open squares). The LMC abundances are from Van der Swaelmen et al. (2013, dark blue open diamonds). The disc abundances are from Bensby, Feltzing \& Oey (2014, light blue open squares), Adibekyan et al. (2012, light blue open circles), and Battistini \& Bensby (2015, light blue open diamonds).

observations, the $[\mathrm{Na} / \mathrm{Fe}]$ ratio decreases with metallicity indicating contributions from SNe Ia, similar to the behaviour of $\alpha$ elements. At $[\mathrm{Fe} / \mathrm{H}] \lesssim-1$ dex, we generally measure $[\mathrm{Na} / \mathrm{Fe}]>0$ dex, while the results from the EMBLA survey generally have $[\mathrm{Na} / \mathrm{Fe}]<0$ dex (Howes et al. 2015). The results from Yong et al. (2013) in the halo show high levels of $[\mathrm{Na} / \mathrm{Fe}]$. However, when they take NLTE into account, their results approach $[\mathrm{Na} / \mathrm{Fe}] \approx 0 \mathrm{dex}$, similar to Roederer et al. (2014). Our results already take NLTE into account and use the same NLTE corrections as Roederer et al. (2014) and Howes et al. (2015). Therefore, it is unlikely that our higher $[\mathrm{Na} / \mathrm{Fe}]$ abundances, with respect to halo observations, are merely a NLTE effect. However, it is possible that differences in analysis methods (e.g. line lists, model atmospheres, etc.) cause systematic offsets between ours and other survey's abundances.

Assuming systematic offsets do not entirely account for the higher $[\mathrm{Na} / \mathrm{Fe}]$ ratio we measure in the bulge compared to the Galactic halo population, we can infer some of the differences in their chemical evolution histories. Given the metallicity dependence of $\mathrm{Na}$ yields from SNe II, where more metal-rich stars have higher yields, our stars must have been enriched by a more metal-rich population than stars of similar metallicity in the Galactic halo. Therefore, our results indicate a short star formation time-scale and rapid enrichment consistent with 

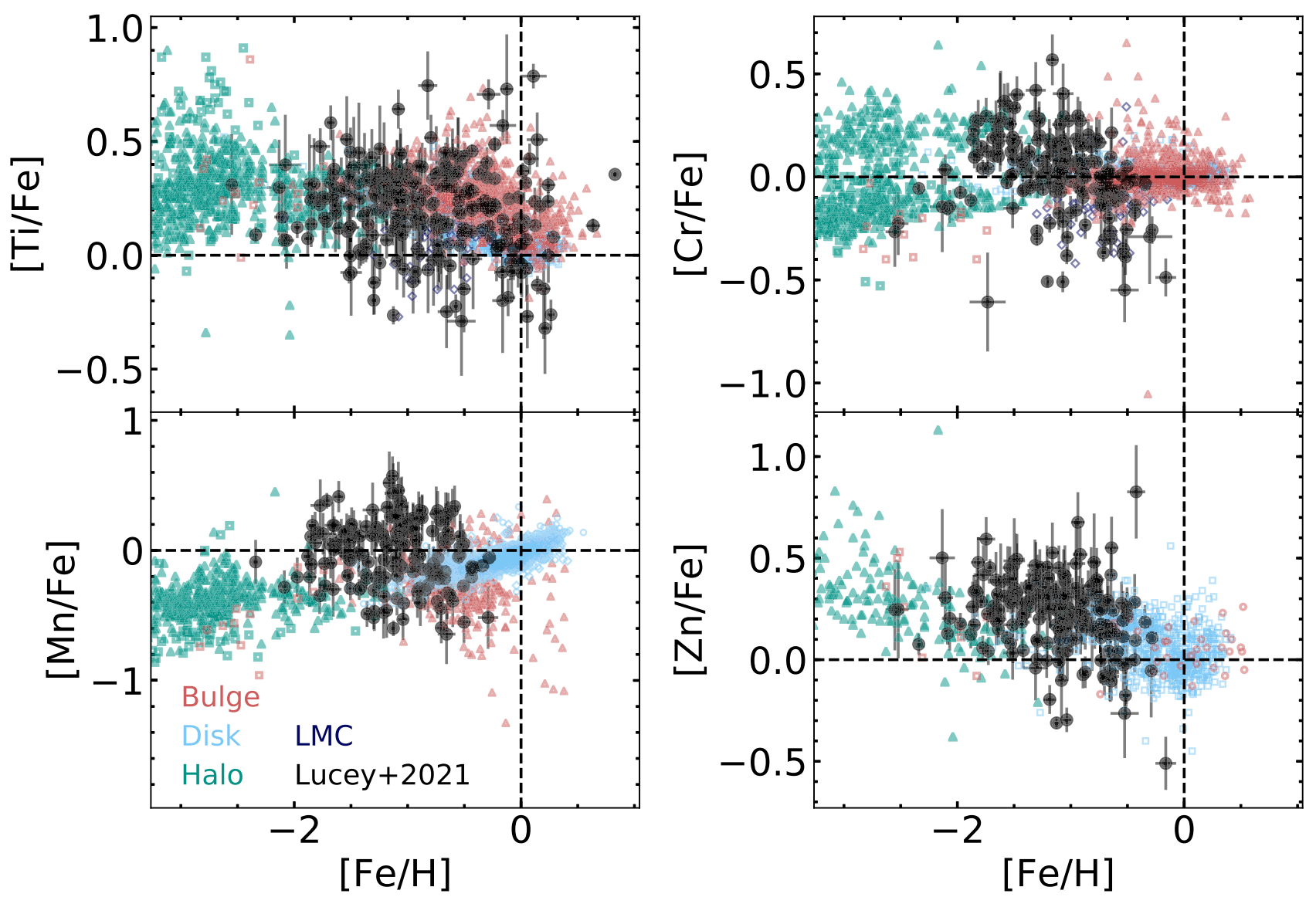

Figure 8. Abundance ratios as a function of metallicity for the Fe-peak elements (Ti, Cr, Mn, and Zn). Symbols are the same as in Fig. 7. However, we also include Zn abundances from Bensby et al. (2017, red open circles).

chemical evolution models for the bulge (e.g. Kobayashi \& Nakasato 2011). However, it is important to note that the Na lines used (4668.6 and $4751.8 \AA$ ) are too weak to measure low Na abundances for metalpoor stars. Therefore, it is possible that our lack of stars with $[\mathrm{Na} / \mathrm{Fe}]$ $<0$ dex at low metallicity is a measurement effect.

\subsubsection{Al}

$\mathrm{Al}$ abundances in the bulge are typically higher than in the disc at $[\mathrm{Fe} / \mathrm{H}] \gtrsim-1$ dex (Bensby et al. 2017; Duong et al. 2019b). However, our $[\mathrm{A} 1 / \mathrm{Fe}]$ abundances are generally consistent with the disc at $[\mathrm{Fe} / \mathrm{H}] \gtrsim-1 \mathrm{dex}$, although they show large scatter. At $[\mathrm{Fe} / \mathrm{H}] \lesssim$ -1 dex, our reported $[\mathrm{Al} / \mathrm{Fe}]$ abundances continue to show a large scatter. However, they are generally higher than abundances from the EMBLA survey (Howes et al. 2015) and the Galactic halo (Yong et al. 2013; Roederer et al. 2014). Unlike our abundances, Howes et al. (2015), Yong et al. (2013), and Roederer et al. (2014) do not perform NLTE line corrections, which may account for some of the offset at low metallicity. Similar to the $\mathrm{Na}$ abundances, the $\mathrm{Al}$ abundances are consistent with a short star formation time-scale and rapid chemical evolution.

The high scatter in the $\mathrm{Al}$ abundances may indicate inhomogeneous mixing or multiple populations. We note that the standard deviation of our $[\mathrm{Al} / \mathrm{Fe}]$ abundances is $\sim 0.51$ dex while the mean uncertainty is $\sim 0.07$ dex. Therefore, it is unlikely that the observed scatter is merely due to uncertainties in the abundances. Interestingly, large $\mathrm{Al}$ enhancement is a signature of second-generation globular cluster stars. This signature has been identified in a couple of stars in this work and will be further discussed in Section 10.

\subsection{Fe-peak elements}

The Fe-peak elements, although formed in a variety of ways, generally trace the $\mathrm{Fe}$ abundance with only small variations (Iwamoto et al. 1999; Kobayashi et al. 2006; Nomoto et al. 2013). However, these slight variations can be extremely informative for supernova physics and chemical evolution models (Kobayashi \& Nakasato 2011). Of the Fe-peak elements, we measure Ti, Cr, Mn, and $\mathrm{Zn}$. We show these results in Fig. 8 compared to other MW populations from the literature.

\subsubsection{Ti}

Frequently considered an $\alpha$-element, Ti is similarly overproduced in $\mathrm{SNe}$ II and underproduced in $\mathrm{SNe}$ Ia with respect to Fe. Therefore, it is expected to behave similarly to the $\alpha$-elements. In the bulge, the $[\mathrm{Ti} / \mathrm{Fe}]$ ratio is generally higher than that in the disc at $[\mathrm{Fe} / \mathrm{H}] \gtrsim-1$ dex (Bensby et al. 2017; Duong et al. 2019a). Our abundances, however, show a large scatter even at high metallicity, with some stars matching the low $\mathrm{Ti}$ abundances observed in the Large Magellanic Cloud (LMC; Van der Swaelmen et al. 2013). This is difficult to draw strong conclusions from given that our 
analysis uses NLTE while the other bulge surveys do not (Bensby et al. 2017; Duong et al. 2019a). None the less, this result suggests that our sample is a mixed stellar population with a variety of origins.

\subsection{2 $\mathrm{Cr}$}

$\mathrm{Cr}$ abundances in the bulge and disc closely follow the Fe abundance (Bensby et al. 2014, 2017; Duong et al. 2019b). This is mostly true for our sample at $[\mathrm{Fe} / \mathrm{H}] \gtrsim-1$ dex, although we observe large scatter and an overabundance of stars with $[\mathrm{Cr} / \mathrm{Fe}]<0$ dex. Interestingly, we observe a number of stars with $[\mathrm{Cr} / \mathrm{Fe}]$ abundance ratios similar to the LMC (Van der Swaelmen et al. 2013). At $[\mathrm{Fe} / \mathrm{H}] \lesssim-1$ dex, the $[\mathrm{Cr} / \mathrm{Fe}]$ ratio decreases with decreasing metallicity similar to results from the EMBLA survey (Howes et al. 2015). It is interesting to note that low $[\mathrm{Cr} / \mathrm{Fe}]$ is inconsistent with chemical enrichment from PISNe.

\subsubsection{Mn}

Mn has a metallicity-dependent yield in SNe II. In general, it is thought to be underproduced with respect to $\mathrm{Fe}$ in $\mathrm{SNe}$ II and overproduced in SNe Ia. Therefore, at $[\mathrm{Fe} / \mathrm{H}] \gtrsim-1 \mathrm{dex}$ in the MW $[\mathrm{Mn} / \mathrm{Fe}]$ increases. This trend is observed in the disc, as shown in Fig. 8. However, this is not observed in our sample or the sample from the HERBS survey (Duong et al. 2019b). Both of these bulge samples show high scatter that is generally centred at $[\mathrm{Mn} / \mathrm{Fe}] \approx 0$ dex, although the number of stars with $[\mathrm{Mn} / \mathrm{Fe}]<0$ dex increases with increasing $[\mathrm{Fe} / \mathrm{H}]$. This result is interesting and likely indicates inhomogeneous mixing of the ISM or that the bulge is made up of multiple stellar populations with different chemical evolution histories. Of the samples shown in Fig. 8, the only work to perform NLTE line corrections is Battistini \& Bensby (2015), shown in light blue open diamonds.

\subsubsection{Zn}

$\mathrm{Zn}$ is produced in core-collapse supernovae with high explosion energy (i.e. hypernovae) and its yields depend strongly on supernova physics. At $[\mathrm{Fe} / \mathrm{H}] \gtrsim-1 \mathrm{dex}$, the $[\mathrm{Zn} / \mathrm{Fe}]$ ratio decreases with increasing metallicity in our sample as well as literature samples for the disc and bulge (Bensby et al. 2014, 2017). This is consistent with yields from SNe Ia. At $[\mathrm{Fe} / \mathrm{H}] \lesssim-1$ dex, our observed $[\mathrm{Zn} / \mathrm{Fe}]$ ratios are consistent with the EMBLA survey (Howes et al. 2015) and the Galactic halo (Roederer et al. 2014). This is also consistent with Galactic chemical evolution models where the large spread in $[\mathrm{Zn} / \mathrm{Fe}]$ is a result of the metallicity and mass-dependent yields from hypernovae (Kobayashi \& Nakasato 2011; Kobayashi, Karakas \& Lugaro 2020).

\subsection{Neutron-capture elements}

Neutron-capture elements are produced through the successive capture of neutrons either through a rapid (r) process or a slow (s) process. In this work, we measure $\mathrm{Ba}$ and $\mathrm{Ce}$ abundances that are thought to be primarily produced through s-processes, specifically in AGB stars. However, they can both be produced in r-process sites as well (Kobayashi et al. 2020). We show the results for these elements in Fig. 9.

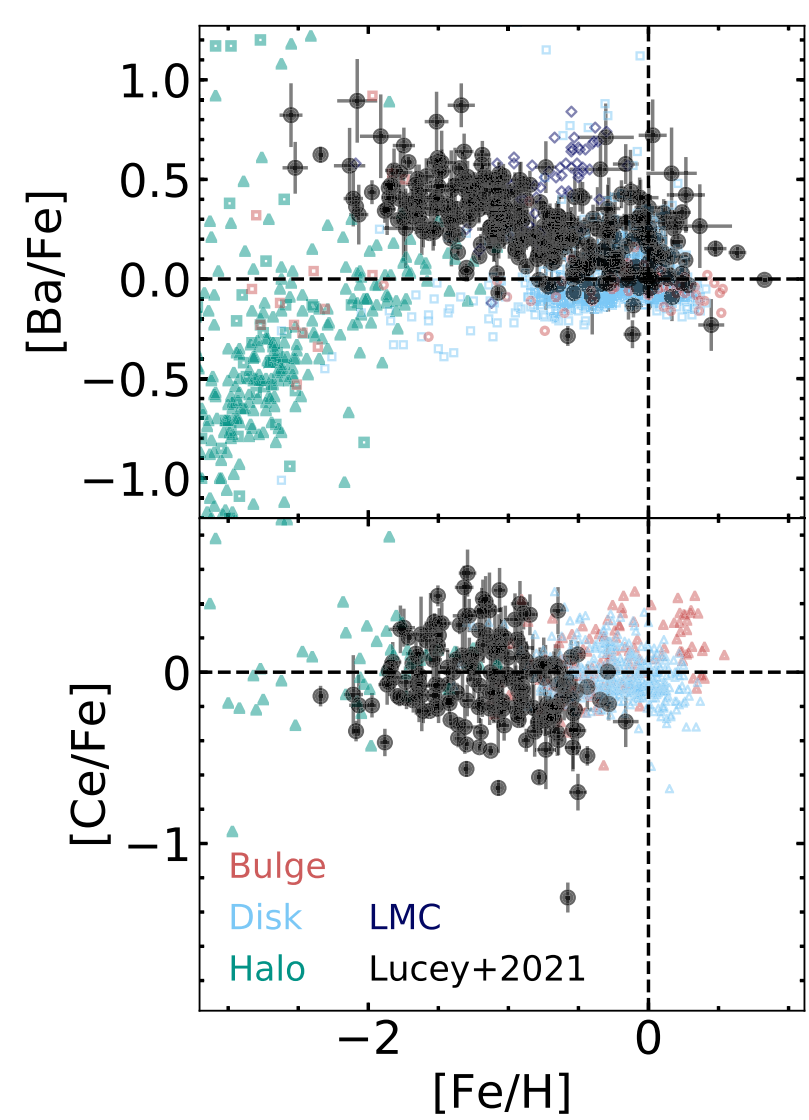

Figure 9. Abundance ratios as a function of metallicity for the neutroncapture elements $\mathrm{Ba}$ and $\mathrm{Ce}$. Symbols are the same as in Figs 7 and 8, with the addition of Ce abundances from Battistini \& Bensby (2016) in red triangles.

\subsubsection{Ba}

Generally, stars in the MW with $[\mathrm{Fe} / \mathrm{H}] \gtrsim-1$ dex show $[\mathrm{Ba} / \mathrm{Fe}]$ ratios that are roughly solar (Bensby et al. 2014, 2017). In the Galactic halo for stars with $[\mathrm{Fe} / \mathrm{H}] \lesssim-1$ dex a large scatter in $[\mathrm{Ba} / \mathrm{Fe}]$ is observed (Yong et al. 2013; Roederer et al. 2014). None the less, the general trend in the halo is $[\mathrm{Ba} / \mathrm{Fe}]$ decreasing with decreasing metallicity. However, r-process events, like electron-capture (EC) supernovae (Truran 1981; Cowan, Thielemann \& Truran 1991), magneto-rotationally driven (MRD) supernovae (Winteler et al. 2012; Nishimura, Takiwaki \& Thielemann 2015), or neutron star mergers (Rosswog et al. 1999), for example, can enhance the $[\mathrm{Ba} / \mathrm{Fe}]$ ratio to values $>1$ dex (Cescutti \& Chiappini 2014).

The EMBLA survey found that most of their metal-poor bulge stars show a decreasing trend of $[\mathrm{Ba} / \mathrm{Fe}]$ with decreasing metallicity, and therefore did not find evidence for an r-process event (Howes et al. 2015). However, our sample shows the opposite trend with the $[\mathrm{Ba} / \mathrm{Fe}]$ ratio increasing at lower metallicities. We note that our survey performs NLTE abundance corrections for Ba while the EMBLA survey does not (Howes et al. 2016). In order to have $[\mathrm{Ba} / \mathrm{Fe}]>0$ and increasing at lower metallicities, it is likely that an r-process event enriched the gas from which these stars formed.

Given the predictions from cosmological simulations that the metal-poor stars in the bulge are ancient (Tumlinson 2010; Kobayashi \& Nakasato 2011; Starkenburg et al. 2017a; El-Badry et al. 2018b), the r-process event that enriched these stars must have occurred on 
Table 1. Properties of the dynamical groups.

\begin{tabular}{|c|c|c|c|c|c|c|c|}
\hline Associated structure & $P($ conf. $)$ & $\begin{array}{c}r_{\text {apo }} \\
(\mathrm{kpc})\end{array}$ & $\begin{array}{l}z_{\max } \\
(\mathrm{kpc})\end{array}$ & Number of stars & Min. $[\mathrm{Fe} / \mathrm{H}]$ & Max. [Fe/H] & Mean $[\mathrm{Fe} / \mathrm{H}]$ \\
\hline Inner bulge & $>0.5$ & & & 136 & -2.52 & 0.37 & -0.92 \\
\hline Halo & $\leq 0.5$ & & $>2.5$ & 32 & -1.97 & 0.01 & -1.09 \\
\hline Disc & $\leq 0.5$ & $>5$ & $\leq 2.5$ & 67 & -2.11 & 0.83 & -0.50 \\
\hline
\end{tabular}

a short time-scale. As neutron star mergers are thought to occur on time-scales $\gtrsim 4 \mathrm{Gyr}$, it is unlikely that our sample received its r-process material from one of these events. MRD SNe have the shortest time-scale, with 1-10 per cent of stars with $10 \mathrm{M}_{\odot} \leq M \leq$ $80 \mathrm{M}_{\odot}$ exploding as MRD SNe (Woosley \& Heger 2006; Winteler et al. 2012; Cescutti \& Chiappini 2014). EC SNe, on the other hand, are thought to occur for all stars with $8 \mathrm{M}_{\odot} \leq M \leq 10 \mathrm{M}_{\odot}$ (Cescutti et al. 2013). Cescutti, Chiappini \& Hirschi (2018) demonstrated that the MRD SN scenario occurs on a fast enough time-scale to enhance $[\mathrm{Ba} / \mathrm{Fe}]$ ratios in metal-poor bulge stars, while the EC SN scenario does not.

\subsubsection{Ce}

In the MW, at all metallicities, Ce tracks the Fe abundance, with the $[\mathrm{Ce} / \mathrm{Fe}] \approx 0$ dex (Roederer et al. 2014; Battistini \& Bensby 2016; Duong et al. 2019b). However, at low metallicities in the Galactic halo, there is large scatter in the $[\mathrm{Ce} / \mathrm{Fe}]$ ratio, similar to $[\mathrm{Ba} / \mathrm{Fe}]$. Unlike $[\mathrm{Ba} / \mathrm{Fe}]$, our stars do not show r-process enhancement in the $[\mathrm{Ce} / \mathrm{Fe}]$ ratio. Given that the r/s-process ratios for $\mathrm{Ba}$ and $\mathrm{Ce}$ are very similar (Simmerer et al. 2004), it is expected that they would be equally enhanced in r-process events and display similar trends with $[\mathrm{Fe} / \mathrm{H}]$. However, unlike $\mathrm{Ba}$, we do not perform NLTE abundance corrections for $\mathrm{Ce}$. Therefore, it is possible that NLTE effects may be obscuring a trend in $[\mathrm{Ce} / \mathrm{Fe}]$ with $[\mathrm{Fe} / \mathrm{H}]$. Future work to measure further NLTE neutron-capture abundances is essential for constraining the chemical enrichment history of the metal-poor bulge.

\section{DYNAMICALLY SEPARATING THE MIXED STELLAR POPULATIONS}

Results from COMBS II demonstrate that metal-poor bulge stars $([\mathrm{Fe} / \mathrm{H}]<-1 \mathrm{dex})$ are comprised of multiple stellar populations that can be separated dynamically. Specifically, COMBS II separated these stars into a population that stays confined to within $3.5 \mathrm{kpc}$ of the Galactic Centre throughout their orbits and those that do not. In this work, we go one step further and divide the unconfined population into multiple dynamically defined groups.

\subsection{Selection method}

In total, we separate our observed stars into four groups. The groups are defined using the probability of confinement $[P($ conf. $)$; see section 4.2 in COMBS II for more details on how this is determined], the apocentre $\left(r_{\text {apo }}\right)$, and the maximum distance from the Galactic plane that the stars reach during their orbit $\left(z_{\max }\right)$. The orbital properties are calculated in COMBS II using GALPY (Bovy 2015). Specifically, we use a Dehnen bar potential (Dehnen 2000) generalized to 3D (Monari et al. 2016) with parameters designed to match the long, slow bar model put forth by Portail et al. (2017). The selection of the dynamical groups is described in Table 1.
Table 2. Chemical complexity of the dynamical groups.

\begin{tabular}{lccc}
\hline $\begin{array}{l}\text { Associated } \\
\text { structure }\end{array}$ & $\begin{array}{c}\text { Mean absolute } \\
\text { correlation } \\
\text { strength }\end{array}$ & $\begin{array}{c}\text { Variance } \\
\text { explained by } \\
\text { four components }\end{array}$ & $\begin{array}{c}\text { Relative } \\
\text { chemical } \\
\text { complexity }\end{array}$ \\
\hline Inner bulge & $0.36 \pm 0.12$ & $96.6 \pm 0.4$ per cent & Highest \\
Outer bulge & $0.55 \pm 0.25$ & $95.0 \pm 1.0$ per cent & - \\
Halo & $0.59 \pm 0.30$ & $98.6 \pm 0.5$ per cent & Lowest \\
\hline
\end{tabular}

Note. We define stellar populations that have higher chemical dimensionality and less correlated abundances as being more chemical complex than populations with lower dimensionality and more highly correlated abundances.

We label the groups based on the Galactic structures to which the majority of the stars belong. However, as with most methods of tagging stars to Galactic structures, there is likely contamination since the structures overlap spatially and kinematically (Carrillo et al. 2020). Our inner bulge population is based on an apocentre cut of $<3.5 \mathrm{kpc}$. However, it is now thought that the bar likely extends out to $5 \mathrm{kpc}$ (Wegg, Gerhard \& Portail 2015). Therefore, we also define an outer bulge population that is likely part of the bulge but does not stay confined to within $3.5 \mathrm{kpc}$ of the Galactic Centre. To separate the outer bulge and halo population, we use a $z_{\max }$ cut of $2.5 \mathrm{kpc}$. This cut is based on the $z_{\max }$ distribution of the inner bulge stars. Given the X-shape of the MW bulge, it is possible that the outer bulge is more flared and reaches larger heights above and below the Galactic plane than in the inner regions. This would cause contamination of the halo population by stars belonging to the bulge. However, after visual inspection of many of the orbits of stars in the halo group, the overwhelming majority have Galactic halo-like orbits and clearly do not belong to the bulge. To separate the outer bulge from the disc population, we use an $r_{\text {apo }}$ cut of $5 \mathrm{kpc}$ based on the proposed length of the bar from Wegg et al. (2015). It is important to note that there is certainly a disc population within $5 \mathrm{kpc}$ of the Galactic Centre that is included in our outer bulge population. However, we are most concerned with simply removing the solar vicinity disc contamination from our sample, rather than selecting bar/bulge stars. Primarily, we aim to compare the metal-poor stars in the innermost region of the Galaxy to the metal-poor stars in the surrounding regions. Therefore, we mostly focus on the inner bulge, outer bulge, and halo populations for the rest of this work.

In Fig. 10, we show the properties of our four groups to confirm that they match expectations for the associated structures. For each group, we apply a Gaussian kernel density estimator (KDE) to the eccentricity and metallicity distributions. The eccentricity distributions of the inner and outer bulge populations are very similar, consistent with the stars being different parts of the same structure. Furthermore, our halo population has highly eccentric orbits with a median eccentricity of $\sim 0.67$. Lastly, the disc is the least eccentric population, which also matches expectations.

We show the MDFs of the dynamically defined groups in the bottom panel of Fig. 10. These distributions are determined using 
KDEs. It is important to note that we do not expect these MDFs to represent the associated structures, given our photometric selection method. None the less, our results generally match expectations for the given structures and selection method. Specifically, we see that our target selection was generally successful and our inner bulge population peaks at $[\mathrm{Fe} / \mathrm{H}] \approx-1 \mathrm{dex}$. However, there is some contamination by metal-rich bulge stars, as the inner bulge distribution also contains a metal-rich peak at $[\mathrm{Fe} / \mathrm{H}] \approx 0$ dex. The outer bulge distribution is very similar to the inner bulge distribution, although the peak's metallicity is slightly higher. In addition, the outer bulge distribution does not have a second metal-rich peak. Although, this is likely a selection effect.

The halo population has the most metal-poor peak, consistent with results from COMBS II, which found that the fraction of halo interlopers increases with decreasing metallicity. However, it is interesting to note the second metal-rich peak. We confirm that these stars have high eccentricity $(e>\sim 0.5)$ and their orbits match expectations for halo stars. However, more precise positional and kinematic data are required to confirm the existence of this metal-rich halo population in the inner Galaxy. The most metal-rich population in our sample is the disc, but it has a large metal-poor tail. This is expected for the disc population, as it is known to have a metal-weak component (Beers et al. 2014; Carollo et al. 2019).

\subsection{Distinct chemical distributions of dynamically defined groups}

In addition to dynamical and metallicity differences, the halo, outer bulge, and inner bulge all show differences in their abundance trends. In Fig. 11, we show the abundance trends for the halo, outer bulge, and inner bulge populations as a function of metallicity for a number of key elements. Specifically, we show the $\alpha$-elements $(\mathrm{Mg}, \mathrm{Si}$, and $\mathrm{Ca})$, one odd-Z element $(\mathrm{Na})$, one Fe-peak element $(\mathrm{Mn})$, and one neutroncapture element $(\mathrm{Ba})$. For each population, the lines shown are the median values, and the error bars correspond to the asymmetric $1 \sigma$ spread. We also show the uncertainty on the median as $\sigma /(N-1)^{0.5}$, where $N$ is the number of stars.

The comparison of $\alpha$-element trends between the halo, outer bulge, and inner bulge populations shows a consistent story. At low metallicities $([\mathrm{Fe} / \mathrm{H}] \lesssim-1 \mathrm{dex})$, the three populations show similar plateau values. However, the outer bulge consistently has the highest median, followed by the halo and then the inner bulge population. It is interesting to note that the difference between the median $\alpha$ abundance trends at the lowest $[\mathrm{Fe} / \mathrm{H}]$ is smallest for $\mathrm{Mg}(\sim 0.1 \mathrm{dex})$ that is a hydrostatic $\alpha$-element, while the explosive $\alpha$-elements, $\mathrm{Si}$ and $\mathrm{Ca}$, have larger differences $(\sim 0.2-0.3 \mathrm{dex})$. At this metallicity, the inner bulge population's median $[\alpha / \mathrm{Fe}]$ is lower than the halo and outer bulge values because of a few stars with especially low $[\alpha / \mathrm{Fe}]$ ratios. It is important to note that the inner bulge population contains stars with $[\alpha / \mathrm{Fe}]$ values as high as the most $\alpha$-enhanced outer bulge stars, while the halo population does not.

The halo population's $[\alpha / \mathrm{Fe}]$ ratio decreases sharply, with the halo becoming the least $\alpha$-enhanced population at $-2 \mathrm{dex} \lesssim[\mathrm{Fe} / \mathrm{H}] \lesssim-1$ dex. This change might be due to the onset of contributions from $\mathrm{SNe}$ Ia. However, the decreasing trend of $[\mathrm{Mn} / \mathrm{Fe}]$ cannot be explained by SN Ia enrichment. This trend continues to higher metallicities, with the halo population generally having lower $[\alpha / \mathrm{Fe}]$ values, indicating a longer star formation duration. On the other hand, the outer and inner bulge populations have very similar $[\alpha / \mathrm{Fe}]$ distributions at high metallicities, indicating similar star formation histories.

To test the statistical significance of the differences in the distributions, we perform 2D Kolmogorov-Smirnov tests (Peacock 1983;
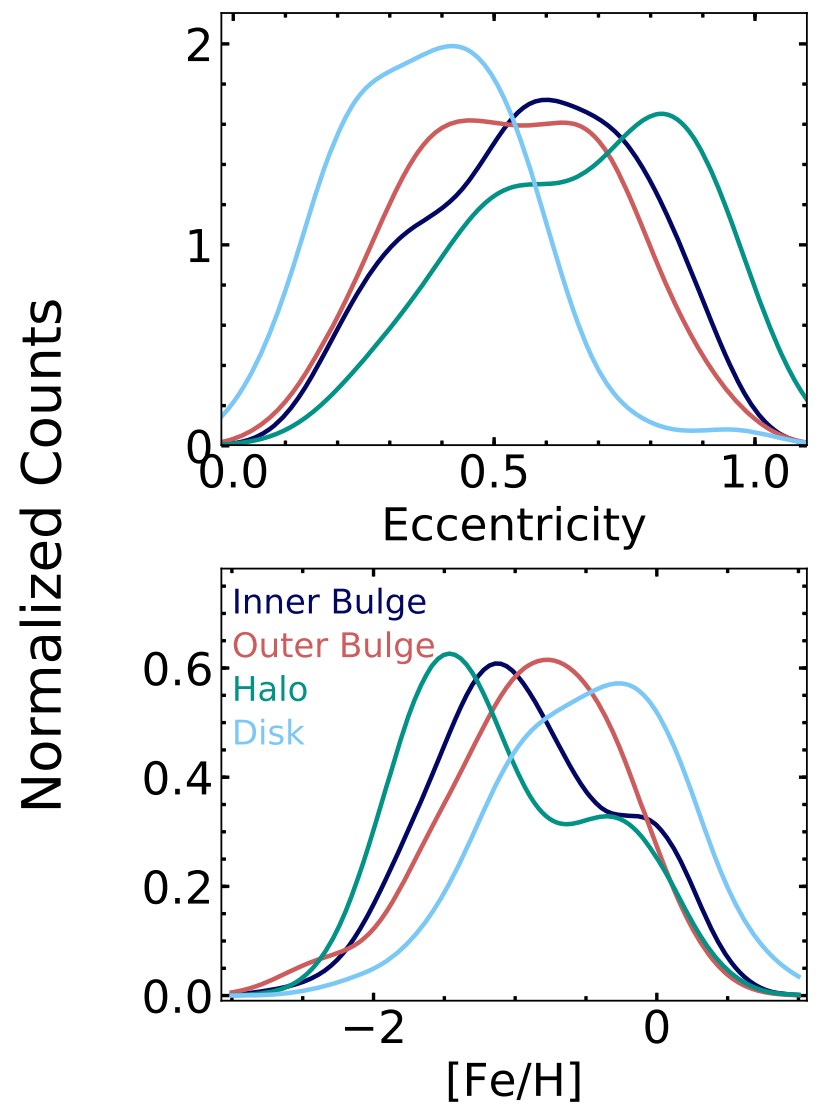

Figure 10. Properties of the populations we associate with various Galactic structures. In the top plot, we show the eccentricity distributions, while the bottom plot shows the MDFs. The inner (136 stars; dark blue) and outer bulges (84 stars; red) have eccentricity distributions consistent with expectations for the MW's bulge. The halo population (32 stars; green) is consistent with highly eccentric halo stars that pass through the Galactic Centre region. The disc population (67 stars; light blue) has an eccentricity distribution consistent with the MW disc. The MDFs are consistent with expectations given our photometric selection method. Namely, the halo is the most metalpoor component and the disc is the most metal rich.

Fasano \& Franceschini 1987). We perform the test 1000 times sampling the abundances from a Gaussian distribution centred on their measured value with a width corresponding to the uncertainty. We then report the mean $p$-value of those 1000 test as our final confidence level. The $[\mathrm{Ca} / \mathrm{Fe}]$ and $[\mathrm{Mg} / \mathrm{Fe}]$ distributions as a function of $[\mathrm{Fe} / \mathrm{H}]$ for the outer bulge are different from the halo distributions to a $>90$ per cent confidence level. However, the inner bulge and halo distributions are not significantly different in $[\mathrm{Ca} / \mathrm{Fe}]$ or $[\mathrm{Mg} / \mathrm{Fe}]$, as they both have large scatter. On the other hand, the differences between $[\mathrm{Si} / \mathrm{Fe}]$ as a function of $[\mathrm{Fe} / \mathrm{H}]$ distributions for the halo compared to both the inner and outer bulge populations are statistically significant to $>90$ per cent confidence. The inner and outer bulge distributions are not significantly different for any $\alpha$-elements.

The only elements for which the differences between the outer and inner bulge populations are statistically significant are Mn and $\mathrm{Na}$, which both have metallicity-dependent yields in SNe II. At low metallicities $([\mathrm{Fe} / \mathrm{H}] \lesssim-1 \mathrm{dex})$, the inner bulge population has lower values in $[\mathrm{Mn} / \mathrm{Fe}]$ and $[\mathrm{Na} / \mathrm{Fe}]$ than the outer bulge population. Therefore, the inner bulge stars were generally enriched by a more metal-poor population than the outer bulge stars. This is consistent 

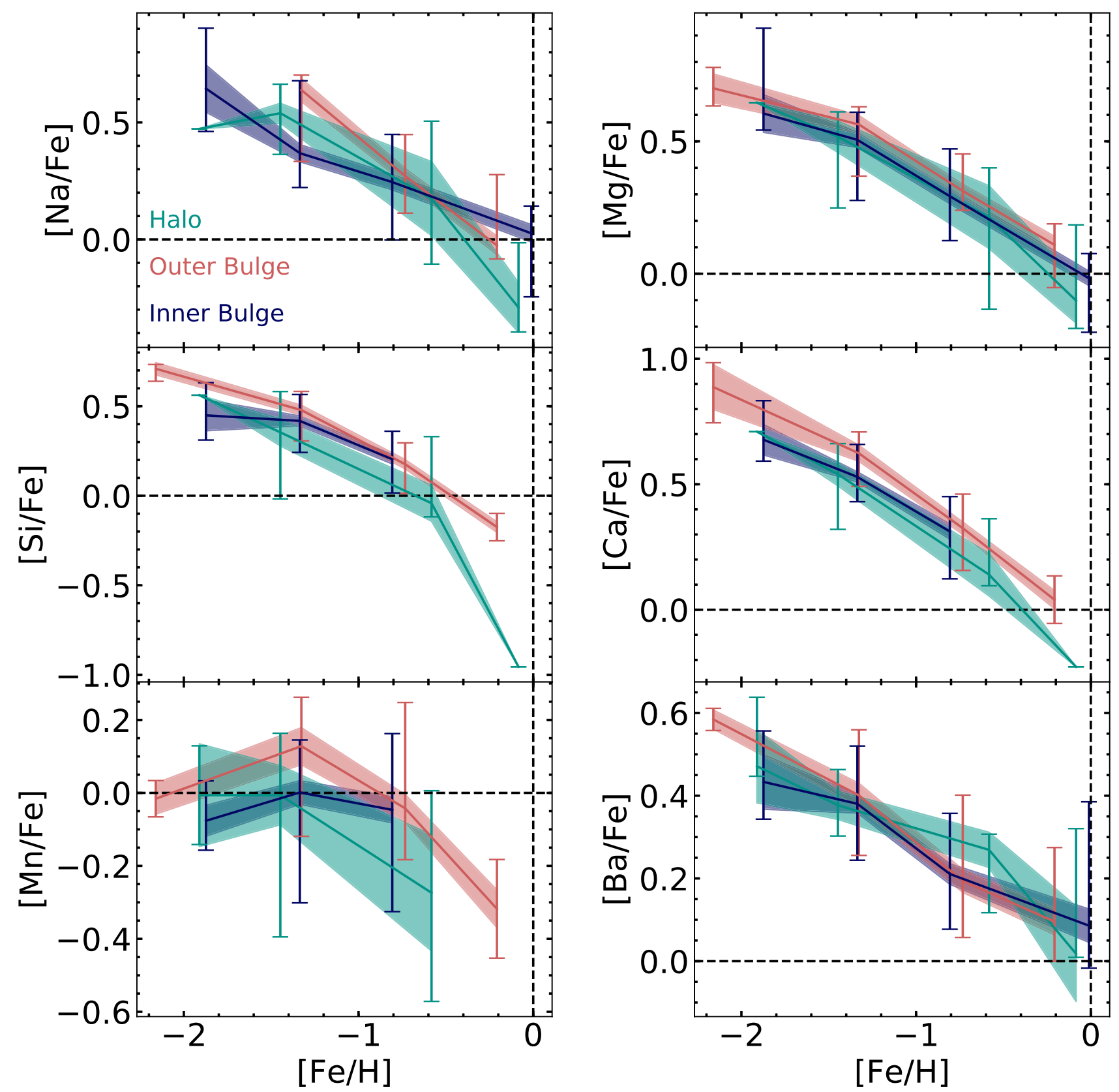

Figure 11. The distributions of a number of key elements as a function of metallicity for the halo (red), outer bulge (green), and inner bulge (dark blue) samples. Specifically, we show the $\alpha$-elements $(\mathrm{Mg}, \mathrm{Si}$, and $\mathrm{Ca}$ ), along with one odd-Z $(\mathrm{Na})$, Fe-peak $(\mathrm{Mn})$, and neutron-capture element (Ba). The lines shown are the median values of the distributions and the error bars correspond to the scatter. We also show the uncertainty on the medians as the shaded regions.

with results from simulations indicating that more tightly bound stars are older than less tightly bound stars of similar metallicity (Tumlinson 2010; El-Badry et al. 2018b).

The difference between $[\mathrm{Mn} / \mathrm{Fe}]$ and $[\mathrm{Na} / \mathrm{Fe}]$ as a function of $[\mathrm{Fe} / \mathrm{H}]$ distributions for the inner bulge and halo populations is not statistically significant. The $[\mathrm{Mn} / \mathrm{Fe}]$ and $[\mathrm{Na} / \mathrm{Fe}]$ distributions for the halo population have large scatter with generally lower values than the outer and inner bulge populations. For $[\mathrm{Mn} / \mathrm{Fe}]$, the halo population starts to decrease at $[\mathrm{Fe} / \mathrm{H}] \sim-1.5 \mathrm{dex}$, consistent with the onset of SNe Ia and a longer star formation time-scale than the inner and outer bulge populations. The difference between $[\mathrm{Mn} / \mathrm{Fe}]$ and $[\mathrm{Na} / \mathrm{Fe}]$ as a function of $[\mathrm{Fe} / \mathrm{H}]$ distributions for the halo compared to the outer bulge population is statistically significant to the $>90$ per cent level.

The $[\mathrm{Ba} / \mathrm{Fe}]$ distributions of the three groups are surprisingly similar to the $[\alpha / \mathrm{Fe}]$ distributions at low metallicity. Specifically, we see the same trend in that the three populations all have similar values at the lowest metallicities, but the outer bulge population has the highest level of enhancement, followed by the halo population and then the inner bulge population. This may indicate that the origin of $\mathrm{Ba}$ in the low-metallicity stars of these populations is similar to the origin of the $\alpha$-elements. However, the similarity to the $[\alpha / \mathrm{Fe}]$ distribution ceases at higher metallicities where the $[\mathrm{Ba} / \mathrm{Fe}]$ ratio for the halo is not significantly lower than that for the inner and outer bulge 
Inner Bulge $-\mathrm{N}=19$
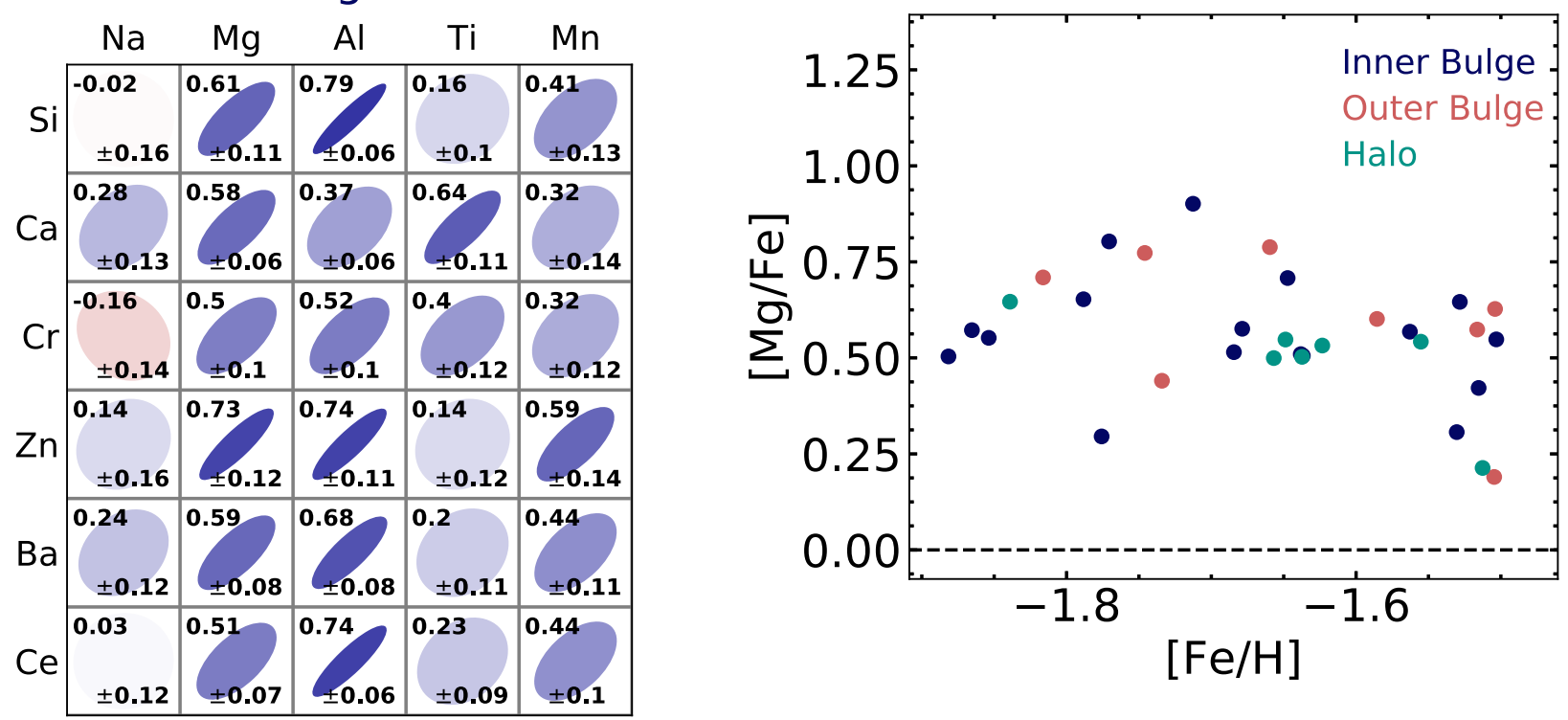

Outer Bulge $-\mathrm{N}=8$

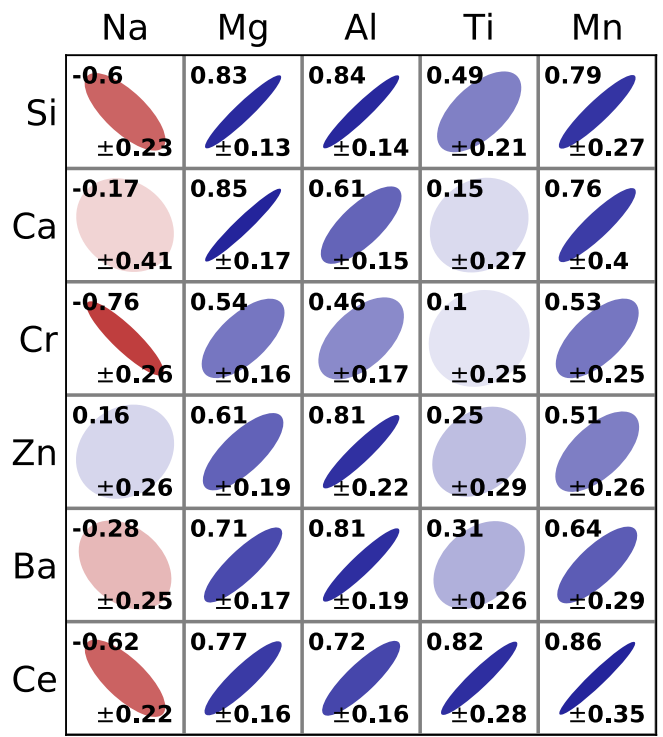

Halo $-\mathrm{N}=8$
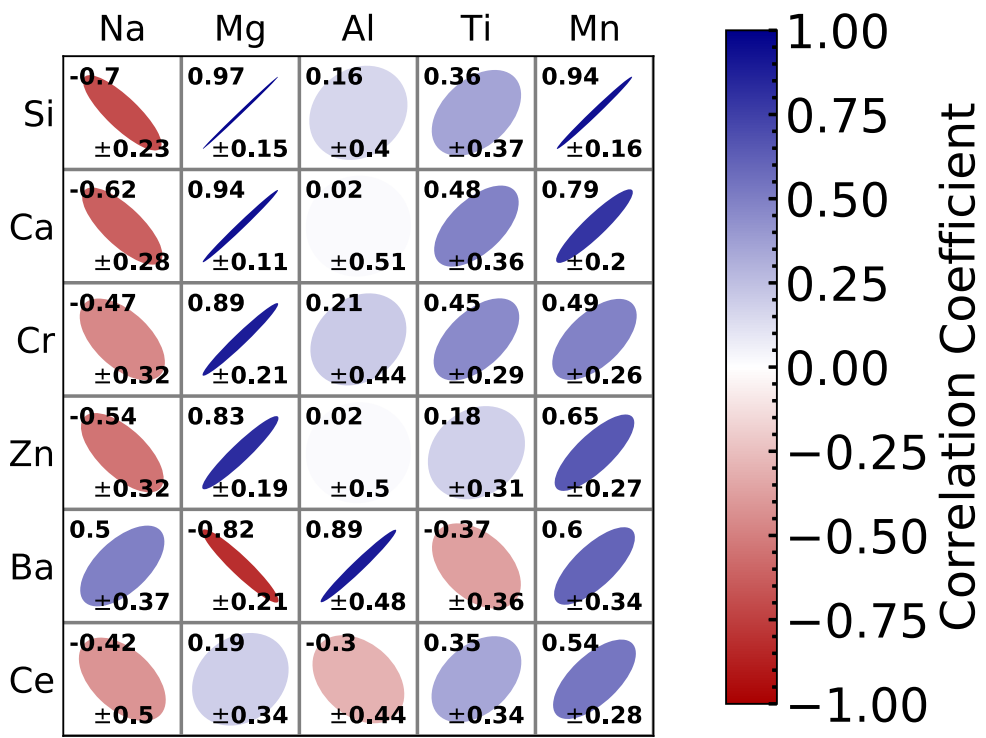

Figure 12. The Pearson correlation coefficients between a number of key elements for the halo (bottom right), outer bulge (bottom left), and inner bulge (top left) populations. Specifically, we compare ( $\mathrm{Na}, \mathrm{Mg}, \mathrm{Al}, \mathrm{Ti}$, and $\mathrm{Mn}$ ) to ( $\mathrm{Si}, \mathrm{Ca}, \mathrm{Cr}, \mathrm{Zn}, \mathrm{Ba}$, and $\mathrm{Ce}$ ). The correlations are calculated using [X/Fe] for stars with $-2 \mathrm{dex} \lesssim[\mathrm{Fe} / \mathrm{H}] \lesssim-1.5 \mathrm{dex}$ and SNR $>40 \mathrm{pixel}^{-1}$. The top right plot shows the $[\mathrm{Mg} / \mathrm{Fe}]$ abundances as a function of $[\mathrm{Fe} / \mathrm{H}]$ for inner bulge (dark blue), outer bulge (green), and halo (red) stars, for reference. To aid in the visualization, we show an oval for each correlation coefficient whose ellipticity, rotation, and colour correspond to the strength and direction of the correlation. We also print the correlation coefficient value in the top left of each correlation box and the corresponding uncertainty on the correlation coefficient in the bottom right. We include the number of stars used in calculating the correlations for the different groups in the title above each correlation plot.

populations. In general, the distribution in $[\mathrm{Ba} / \mathrm{Fe}]$ is much more scattered in the inner and outer bulge populations than in the halo. The differences between the halo distribution of $[\mathrm{Ba} / \mathrm{Fe}]$ as a function of $[\mathrm{Fe} / \mathrm{H}]$ and the outer bulge distribution are statistically significant to the $>90$ per cent confidence level. On the other hand, the outer and inner bulge populations show strikingly similar distributions in $[\mathrm{Ba} / \mathrm{Fe}]$ as a function of $[\mathrm{Fe} / \mathrm{H}]$. This is similar to the $\mathrm{Ca}$, and $\mathrm{Mg}$ abundances, providing further evidence that the halo population has a significantly different chemical evolution history than the outer bulge population.
8.3 Chemical complexity of inner and outer bulges compared to halo population

In addition to the individual elemental distributions, we also study the correlation between elements and the chemical dimensionality of the inner bulge, outer bulge, and halo populations. Through this analysis, we shed light on the diversity of nucleosynthetic events that enriched each population.

In Fig. 12, we show the Pearson correlation coefficients for pairs of a number of key elements in the inner bulge, outer bulge, and halo populations. Specifically, we calculate the correlation coefficient for 
the $[\mathrm{X} / \mathrm{Fe}]$ values, comparing $(\mathrm{Na}, \mathrm{Mg}, \mathrm{Al}, \mathrm{Ti}$, and $\mathrm{Mn})$ to $(\mathrm{Si}, \mathrm{Ca}$, $\mathrm{Cr}, \mathrm{Zn}, \mathrm{Ba}$, and $\mathrm{Ce}$ ). The correlation coefficients are calculated using stars with SNR $>40$ pixel $^{-1}$ and $-2 \mathrm{dex}<[\mathrm{Fe} / \mathrm{H}]<-1.5 \mathrm{dex}$, in order to isolate yields from core-collapse supernovae and limit the impact of metallicity on the correlations. In the top left, we show the results for the inner bulge population that uses 19 stars, while the outer bulge is shown in the bottom left, using 8 stars. We also show results for the halo population on the bottom right, using eight stars. The top right plot shows $[\mathrm{Mg} / \mathrm{Fe}]$ as a function of $[\mathrm{Fe} / \mathrm{H}]$ for the stars used in the correlation plots, as a reference. This figure demonstrates that the stars used for the halo (red), outer bulge (green), and inner bulge (dark blue) populations span similar metallicity ranges. In the three correlation plots, each small box contains an ellipse whose eccentricity and colour correspond to the strength of the correlation. In addition, we print the correlation coefficient in the top right corner, with the corresponding uncertainty on the coefficient in the bottom right corner.

The uncertainties are calculated using a bootstrap method in order to propagate the impact of the abundance uncertainties and the limited number of stars. To account for the abundance uncertainties, we recalculate the correlation coefficient 1000 times with new abundance values each time. These values are randomly selected from Gaussian distributions that are centred on the measured abundance value with a width equivalent to the uncertainty. We then define the correlation coefficient's uncertainty due to abundance uncertainties as the median of the differences between the original correlations and the recalculated values. Similarly, to account for the limited number of stars, we recalculate the coefficients $N$ times dropping out one star from the sample each time. Again, we use the median of the differences between the original correlations and the recalculated values as the uncertainty due to the limited number of stars. We then add the uncertainties due to the number of stars and the uncertainties due to the abundance uncertainties in quadrature for our total uncertainty values. We note that the uncertainties due to the abundance uncertainties are dominant with the uncertainties due to the number of stars being of the order of $\sim 0-0.01$.

Overall, the inner bulge population shows the weakest correlations, followed by the outer bulge population and then the halo. As they are all $\alpha$-elements, it is expected for $\mathrm{Mg}, \mathrm{Si}$, and $\mathrm{Ca}$ to be tightly correlated. This is observed in the halo population, but the correlations are weaker in the outer and inner bulge populations. This is especially interesting given that PISN yields have $[\mathrm{Ca} / \mathrm{Mg}]$ and $[\mathrm{Si} / \mathrm{Mg}]$ abundance ratios that are mass dependent. Therefore, yields from PISNe of varying masses would cause the correlation between $\mathrm{Mg}, \mathrm{Si}$, and $\mathrm{Ca}$ to weaken and become noisier as seen in the inner and outer bulge populations.

Furthermore, the correlation of $\mathrm{Ca}$ and $\mathrm{Si}$ with $\mathrm{Mn}$ in the halo is strikingly strong. This is surprising given that $\mathrm{Mn}$ is thought to have metallicity-dependent yields in SNe II while Si and Ca do not. This may indicate that the halo stars were enriched by a population with a narrow metallicity range. This correlation becomes sequentially weaker as we move to more tightly bound stars in the outer and inner bulge populations. In general, the inner bulge population only shows weak correlations with $\mathrm{Mn}$. In addition, the negative correlation of $\mathrm{Na}$ with $\mathrm{Si}, \mathrm{Ca}$, and $\mathrm{Zn}$ is significant in the halo, but almost completely disappears in the outer bulge population and is non-existent in the inner bulge population. Similar results are found for the positive $\mathrm{Na}$ to Ba correlation.

Excluding Al, which is discussed later, the mean of the absolute correlations of the abundance pairs shown in Fig. 12 is $0.59 \pm 0.30$ for the halo population, while the means for the outer and inner bulge populations are $0.55 \pm 0.25$ and $0.36 \pm 0.12$, respectively.
The uncertainties on the mean are determined by recalculating the mean using the correlation strengths with the individual correlation uncertainties added/subtracted. Therefore, we find evidence that the elemental abundances in the halo population are generally more correlated than in similar metallicity stars in the inner bulge populations. This result may indicate a less diverse chemical enrichment history in the halo population as compared to the inner bulge.

Furthermore, the abundance pairs that do not follow the above trend can provide interesting insight into the possible differences between the chemical enrichment histories of these populations. For example, the $\mathrm{Al}$ abundances show the opposite trend in that the inner and outer bulge populations have strong correlations while the $\mathrm{Al}$ abundances for the halo population are generally not correlated with any elements, except for $\mathrm{Ba}$. This is especially difficult to interpret given that $\mathrm{Na}$ and $\mathrm{Al}$ are thought to be produced in similar ways, but the inner bulge population does not show strong correlations for $\mathrm{Na}$ with any elements. This result solicits further investigation into possible nucleosynthetic sites, beyond $\mathrm{SNe}$ II, for $\mathrm{Al}$ in the inner bulge population.

Another striking difference between the outer bulge, inner bulge, and halo populations is the strength of the positive $\mathrm{Mg}, \mathrm{Ba}$, and Ce correlations in the outer and inner bulge populations, while the halo population shows negative or weak correlations. This is further evidence for the similar origin of $\mathrm{Ba}$ and $\alpha$-elements at low metallicities in the inner and outer bulge populations. Specifically, this result further supports MRD SNe as the origin for $\mathrm{Ba}$ in these ancient stars (Kobayashi et al. 2020). MRD SNe produce high levels of $\mathrm{Ba}$ and $\mathrm{Ce}$ as well as $\alpha$-elements (Yong et al. 2013). However, further work analysing and comparing MRD SNe theoretical yields to the observed abundances is required.

$\mathrm{Ti}$ is another interesting case that does not match the general trend of strong correlations in the halo and weak correlations in the inner bulge population. Specifically, Ti generally does not have significantly strong correlations with any element except for with $\mathrm{Ca}$ in the inner bulge population. We note that the outer bulge shows a somewhat strong correlation between $\mathrm{Ti}$ and $\mathrm{Ce}$; however, the uncertainty is large at 0.28 . It is possible that the $\mathrm{Ca}$ and $\mathrm{Ti}$ correlation in the inner bulge population is insignificant, but it may also indicate an interesting origin for some of the $\mathrm{Ca}$ in this population.

In addition to the correlation analysis, we perform a chemical dimensionality analysis to further explore the differences in the inner bulge, outer bulge, and halo populations. Specifically, we perform principal component analysis (PCA) on the elemental abundances for each population. Essentially, PCA sequentially finds orthogonal components that explain the most variance in the given data. For the analysis, we include all stars in each population with SNR $>40$ pixel $^{-1}$, $[\mathrm{Fe} / \mathrm{H}]<-1$ dex, and a complete set of elemental abundances for $\mathrm{Na}$, $\mathrm{Mg}, \mathrm{Al}, \mathrm{Si}, \mathrm{Ca}, \mathrm{Ti}, \mathrm{Cr}, \mathrm{Mn}, \mathrm{Zn}, \mathrm{Ba}$, and Ce. Similar to the correlation analysis, we choose to only focus on metal-poor stars $([\mathrm{Fe} / \mathrm{H}]<-1$ dex) in this analysis to limit the impact of SNe Type Ia. However, since the PCA analysis requires a complete set of abundances, we are left with only 7 stars from the halo population, 11 stars from the outer bulge population, and 20 stars from the inner bulge population, even though the metallicity range used is larger than for the correlation analysis. To account for the uncertainties in the abundances, we perform the PCA analysis 1000 times with new abundances sampled from a normal distribution centred on the measured abundance with a width corresponding to the abundance uncertainty.

To explore the comparative dimensionality of the elemental abundances, we investigate the percentage of variance explained by each component derived from the PCA. Consistently, we find that for the same number of components, a higher percentage of the 
variance in the halo population is explained compared to the inner and outer bulge populations. For example, $92.2 \pm 1.7$ per cent of the variance is explained by two components in the halo population while only $85.7 \pm 2.6$ and $91.3 \pm 1.0$ per cent is explained in the outer and inner bulge populations, respectively. Furthermore, when using four components, $98.6 \pm 0.5$ per cent of the variance in the halo is explained, while only $95.0 \pm 1.0$ and $96.6 \pm 0.4$ per cent of the variance is explained in the outer and inner bulge populations, respectively. Therefore, we find evidence that the halo population has lower chemical dimensionality than the inner and outer bulge populations.

To describe the combination of our correlation and dimensionality analysis, we define a new term: chemical complexity. In total, we find that the elemental abundances in the halo population are highly correlated with a mean correlation of $0.59 \pm 0.30$, while the mean for the outer and inner bulge populations is $0.55 \pm 0.25$ and $0.36 \pm 0.12$, respectively. Furthermore, we found that the halo population has lower chemical dimensionality than the inner and outer bulge populations. Specifically, when using four components, only $96.6 \pm 0.4$ and $95.0 \pm 1.0$ per cent of the variance in the elemental abundances is explained for the inner and outer bulge populations while the same is true for $98.6 \pm 0.5$ per cent of the elemental abundance variance in the halo population. Therefore, we describe the highly correlated, lower dimensional halo population as less chemically complex compared to the inner and outer bulge populations whose elemental abundances are less correlated and have higher dimensionality. This measure of relative chemical complexity is indicative of the diversity of chemical enrichment events. Therefore, we suggest that the inner and outer bulge populations have a higher diversity of enrichment events compared to the halo population. However, it is important to note that these results may also be impacted by the rate of mixing in the ISM at the different formation times. Specifically, higher chemical complexity could also indicate a less well-mixed ISM.

In total, we discover a number of key results from our comparison between the abundances of the inner bulge, outer bulge, and halo populations, which are summarized in Table 2. First, we find that the inner and outer bulge populations have shorter star formation time-scales and more rapid chemical evolution than the halo population. In addition, our results solicit further investigation into the nucleosynthetic origins of $\mathrm{Ba}$ and $\mathrm{Al}$ in metal-poor inner bulge stars. Furthermore, we find that the abundances are consistent with the inner bulge being the oldest population, compared to the outer bulge and halo populations. We also find that at low metallicity, the inner bulge is the most chemically complex population, followed by the outer bulge and then halo population. Combined, these results suggest that older bulge populations are more chemically complex. This may be due to a combination of diversity of chemical enrichment events (e.g. PISNe, EC SNe, MRD SNe, and other SN predictions for Population III stars), as well as inhomogeneous mixing of the ISM.

\section{PAIR-INSTABILITY SUPERNOVA SIGNATURES}

PISNe are highly energetic thermonuclear explosions that occur after the hydrodynamical collapse caused by electron-positron pair production in massive $\left(>25 \mathrm{M}_{\odot}\right)$ CO cores (Barkat, Rakavy \& Sack 1967; Rakavy, Shaviv \& Zinamon 1967). It is predicted that $\sim 25$ per cent of the first stars would explode as PISNe (Hirano et al. 2015). Therefore, it is expected that $\sim 1 / 400$ stars with $[\mathrm{Ca} / \mathrm{H}]$ $<-2$ dex would be enriched by a PISN (Takahashi et al. 2018). However, chemical signatures of PISNe in studies of metal-poor

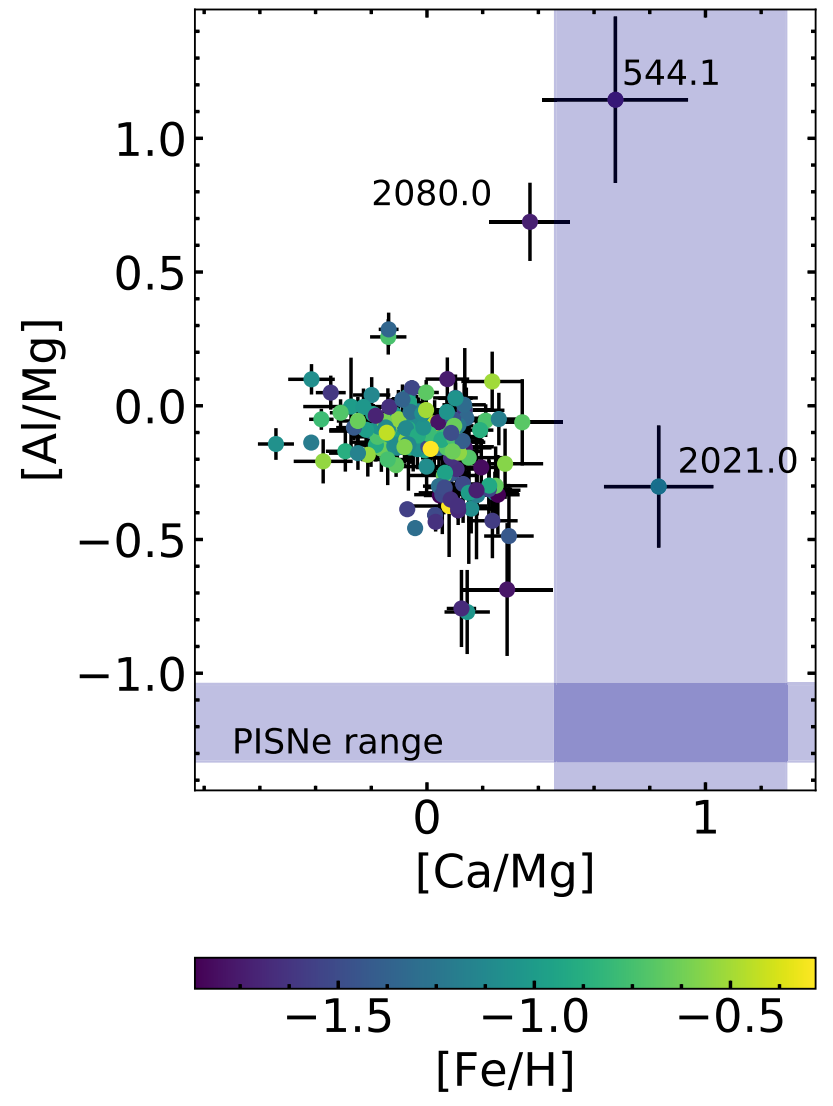

Figure 13. The $[\mathrm{Al} / \mathrm{Mg}]$ ratios as a function of $[\mathrm{Ca} / \mathrm{Mg}]$ for our sample stars, coloured by $[\mathrm{Fe} / \mathrm{H}]$. We shade the regions corresponding to simulated PISN yields (Takahashi et al. 2018). We have two stars (544.1 and 2021.0) with $[\mathrm{Ca} / \mathrm{Mg}]$ ratios consistent with PISN predictions, but their $[\mathrm{Al} / \mathrm{Mg}]$ ratios are significantly higher than predictions.

stars have been elusive. A number of candidates have been put forward, but none perfectly match the predicted abundance trends from simulations (Takahashi et al. 2018). Whether the lack of PISNe chemical signature detections is an observational effect or the result of incorrect simulated rates and yields is yet to be determined.

In Fig. 13, we plot our sample's abundances with respect to the predicted PISN abundance trends from Takahashi et al. (2018). Specifically, we plot abundance ratios that are thought to be especially discriminatory in PISN yields: $[\mathrm{Al} / \mathrm{Mg}]$ and $[\mathrm{Ca} / \mathrm{Mg}]$. The range of predicted PISN abundance yield ratios is shown in the blue-shaded regions. The measured abundances of our sample are shown in points coloured by their metallicity, with corresponding uncertainties as black error bars. We discover two stars that have $[\mathrm{Ca} / \mathrm{Mg}]$ ratios consistent with predictions for PISN yields. However, neither of these stars has a consistent $[\mathrm{Al} / \mathrm{Mg}]$ ratio. Out of the two stars $(544.1$ and 2021.0) with high enough $[\mathrm{Ca} / \mathrm{Mg}]$ ratios, we focus on 2021.0 that has an $[\mathrm{Al} / \mathrm{Mg}]$ ratio closest to PISN yield predictions.

Star 2021.0 has $[\mathrm{Fe} / \mathrm{H}]=-1.07 \mathrm{dex}$ and $[\mathrm{Ca} / \mathrm{H}]=0.05 \mathrm{dex}$. We show all measured abundances for this star in Fig. 14. Specifically, we show $[\mathrm{Mg} / \mathrm{Mg}],[\mathrm{Al} / \mathrm{Mg}],[\mathrm{Si} / \mathrm{Mg}],[\mathrm{Ca} / \mathrm{Mg}]$, and $[\mathrm{Fe} / \mathrm{Mg}]$. We also attempted to measure the other elements presented in this work, but the HR06 spectrum has only SNR $=21$ pixel $^{-1}$, making many of the elements difficult to measure reliably. In Fig. 14, we also plot predicted PISN yields for non-rotating models with various initial masses from Takahashi et al. (2018). Our $[\mathrm{Ca} / \mathrm{Mg}]$ and $[\mathrm{Fe} / \mathrm{Mg}]$ ratios match predictions for PISNe, but our $[\mathrm{Al} / \mathrm{Mg}]$ and $[\mathrm{Si} /(\mathrm{Mg}, \mathrm{Ca}$, 


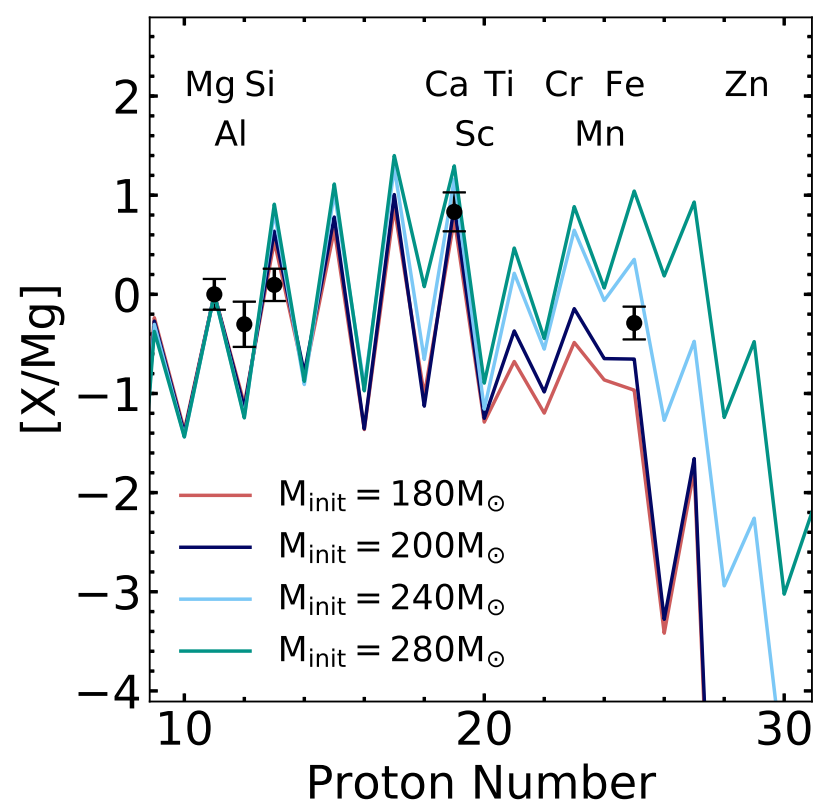

Figure 14. The $[\mathrm{X} / \mathrm{Mg}]$ ratios for star 2021.0 compared to non-rotating model PISN yields of various initial masses from Takahashi et al. (2018). We are only able to measure $\mathrm{Mg}, \mathrm{Al}, \mathrm{Si}, \mathrm{Ca}$, and $\mathrm{Fe}$ for this star as it has $\mathrm{SNR}=21 \mathrm{pixel}^{-1}$. The $[\mathrm{Ca} / \mathrm{Mg}]$ and $[\mathrm{Fe} / \mathrm{Mg}]$ ratios match PISN signatures, but the $[\mathrm{Al} / \mathrm{Mg}]$ and $[\mathrm{Si} / \mathrm{Mg}]$ ratios do not.

$\mathrm{Fe})]$ ratios do not. In fact, our measured $[\mathrm{Si} /(\mathrm{Mg}, \mathrm{Fe})]$ ratio is rather consistent with normal core-collapse supernovae. Furthermore, the metallicity of 2021.0 is higher than expectations for a Population II star that was enriched solely by a single PISN (Karlsson et al. 2008). Therefore, it is possible that this star was enriched by a PISN along with an SN II. However, further observations are needed to measure more elemental abundances in this star to confirm the PISN signature. It is also important to note that that star 2021.0 is part of the inner bulge population with a $P$ (conf. $)=0.93$. Furthermore, star 2021.0 is tightly bound with a pericentre of $0.49 \mathrm{kpc}, r_{\text {apo }}=2.31 \mathrm{kpc}$, and $z_{\max }$ $=1.15 \mathrm{kpc}$. Therefore, it is likely that this star formed in the first few Gyr of star formation in the Universe. However, asteroseismology is required to further constrain its age.

\section{GLOBULAR CLUSTER ORIGIN}

Recent work suggests that the metal-poor bulge may be at least partially built up by dissipated globular clusters (Shapiro, Genzel \& Förster Schreiber 2010; Kruijssen 2015; Bournaud 2016). To date, a significant number of stars in the bulge with chemistry consistent with globular clusters have been detected (Fernández-Trincado et al. 2017; Schiavon et al. 2017; Lucey et al. 2019). Specifically, the chemical signatures encountered include nitrogen enhancement and the $\mathrm{Al}-\mathrm{Mg}$ and $\mathrm{Na}-\mathrm{O}$ anticorrelations that are signatures of secondgeneration globular cluster stars (Gratton, Sneden \& Carretta 2004). However, the rate at which these stars occur among metal-poor bulge stars and whether they are confined stars as opposed to interloping halo stars are yet to be determined.

In this work, we find two stars (544.1 and 2080.0) that have enhanced $\mathrm{Al}$ with respect to their $\mathrm{Mg}$ abundances. In Fig. 15, we show the $[\mathrm{Al} / \mathrm{Fe}]$ abundances as a function $[\mathrm{Mg} / \mathrm{Fe}]$ for our sample. The confined bulge population is shown as black points while the unconfined stars are shown as black crosses. We also show a number of surveys from the literature for comparison. Specifically, we show

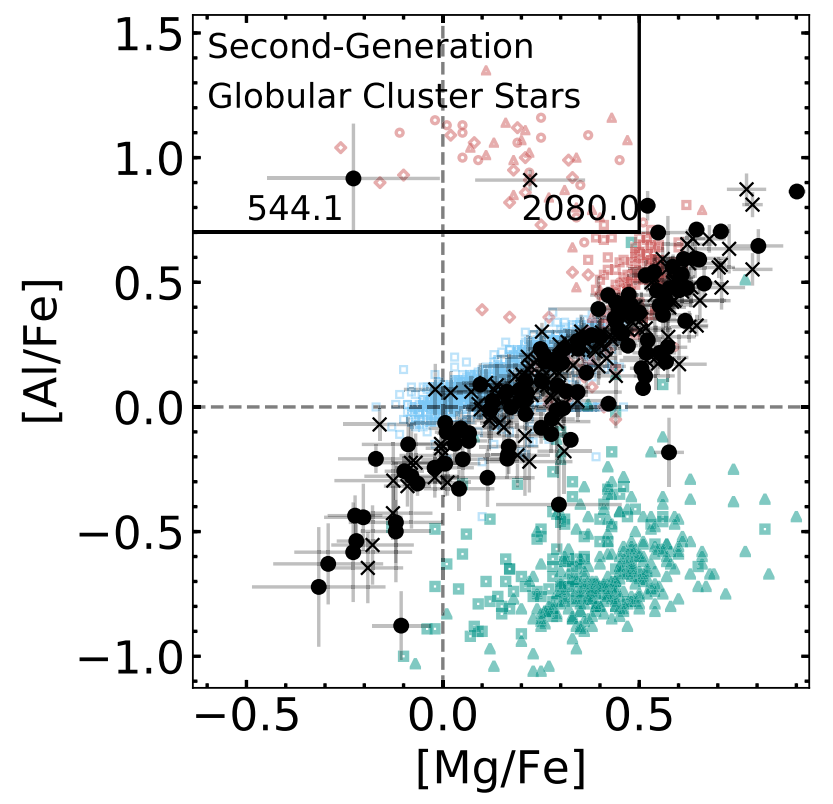

Figure 15. The $[\mathrm{Al} / \mathrm{Fe}]$ abundance ratios as a function of $[\mathrm{Mg} / \mathrm{Fe}]$ for our sample. We show confined bulge stars $[P($ conf. $)>0.5]$ as black points and unconfined $[P($ conf. $) \leq 0.5]$ stars as black crosses. We also show halo (Roederer et al. 2014; Yong et al. 2013, green open triangles and squares, respectively), disc (Bensby et al. 2014, light blue open squares), and globular cluster literature samples for comparison. Specifically, we show NGC 4833 (red open circles), NGC 7089 (red open triangles), and NGC 2808 (red open diamonds) from Pancino et al. (2017), along with NGC 6121 (red open squares) from Marino et al. (2008). We have two stars (544.1 and 2080.0) that have chemistry consistent with second-generation globular cluster stars.

halo samples from Roederer et al. (2014, green open triangles) and Yong et al. (2013, green open squares), along with a disc sample from Bensby et al. (2014, light blue open squares). In addition, we show abundances from the globular clusters NGC 4833 (red open circles), NGC 7089 (red open triangles), and NGC 2808 (red open diamonds) from Pancino et al. (2017), along with NGC 6121 (red open squares) from Marino et al. (2008). The stars 544.1 and 2080.0 match trends seen in globular clusters and have much higher $[\mathrm{Al} / \mathrm{Fe}]$ ratios compared to other stars in our sample with similar $[\mathrm{Mg} / \mathrm{Fe}]$. Star 2080.0 is unconfined and belongs to the disc dynamical group. However, its orbit is unusual for a disc star, with a pericentre of $0.19 \mathrm{kpc}, r_{\text {apo }}=8.6 \mathrm{kpc}$, and $z_{\max }=0.90 \mathrm{kpc}$. On the other hand, star 544.1 is on a typical inner bulge orbit with a pericentre of $0.77 \mathrm{kpc}$, $r_{\text {apo }}=2.79 \mathrm{kpc}$, and $z_{\max }=1.95 \mathrm{kpc}$. It is especially interesting to note that 544.1 specifically matches abundance trends from NGC 2808, one of the MW's most massive globular clusters, that is theorized to be part of the Gaia-Enceladus system (Myeong et al. 2018).

Schiavon et al. (2017) and Horta et al. (2021) estimate that $\sim 25$ per cent of the stellar mass in the inner $2 \mathrm{kpc}$ of the Galaxy is disrupted globular cluster stars, assuming that nitrogen-rich stars are second-generation globular cluster stars. Given these results, it is expected that more than 2 out of our 241 stars with $\mathrm{Al}$ and $\mathrm{Mg}$ measurements would be second-generation globular cluster stars and would therefore show the $\mathrm{Al}-\mathrm{Mg}$ anticorrelation. However, it is unclear whether all second-generation globular cluster stars can be detected using the Al-Mg anticorrelation. To perform apples-toapples comparison, further observations are required to determine the fraction of N-rich stars in our sample. Another possible explanation for the apparent lack of second-generation globular cluster stars in our sample could be the result of SkyMapper photometry for target 
selection. It is known that photometric selection of metal-poor stars using SkyMapper can be biased against selecting C-enhanced stars (Da Costa et al. 2019). As nitrogen-rich stars can frequently be $\mathrm{C}$ rich (Horta et al. 2020), we may be biased against selecting nitrogen-rich stars and therefore second-generation globular cluster stars as well. Last, our results could be discrepant with estimates from Schiavon et al. (2017) and Horta et al. (2021) due to their assumption that all $\mathrm{N}$-rich stars are disrupted globular cluster stars that would lead to an overestimate of the contribution of dissipated globular clusters to the stellar mass. Other origins for $\mathrm{N}$-rich stars have been suggested (e.g. Bekki 2019), which could explain the high fraction of N-rich stars in the inner Galaxy.

\section{SUMMARY AND CONCLUSIONS}

The Galactic bulge is a complex structure with overlapping stellar populations that can provide crucial information about the formation and evolution of the MW. The metal-poor population in the bulge is of special interest given that cosmological simulations predict that they are some of the oldest stars in the Galaxy (Salvadori et al. 2010; Tumlinson 2010; Kobayashi \& Nakasato 2011; Starkenburg et al. 2017a; El-Badry et al. 2018b). However, this population has historically been difficult to study given that it compromises only $\sim 5$ per cent of bulge stars. Now, with the recent advent of metallicitysensitive photometric surveys (Starkenburg et al. 2017b; Wolf et al. 2018; Casagrande et al. 2019), we can target metal-poor stars in the Galactic bulge and study them in large numbers (Arentsen et al. 2020b). Recent work has determined that many metal-poor stars found in the bulge are actually halo interloping stars (Kunder et al. 2020; Lucey et al. 2020). Therefore, it is necessary to combine dynamical and chemical information to disentangle the metal-poor bulge population and study its origins in detail.

In this work, we successfully target metal-poor stars in the Galactic bulge using SkyMapper photometry and observe 555 stars with the VLT/GIRAFFE spectrograph. We report stellar parameters and abundances for 319 that have SNR $>20$ pixel $^{-1}$ and astrometry from Gaia DR2. The stellar parameters and abundances are determined using a $\chi^{2}$ fit to model spectra synthesized using SME with NLTE departure coefficients for $\mathrm{Li}, \mathrm{O}, \mathrm{Na}, \mathrm{Mg}, \mathrm{Al}, \mathrm{Si}, \mathrm{Ca}, \mathrm{Ti}, \mathrm{Fe}$, and $\mathrm{Ba}$ (Valenti \& Piskunov 1996; Piskunov \& Valenti 2017). We compare our stellar parameters to results for GBSs (Jofré et al. 2014; Heiter et al. 2015; Hawkins et al. 2016), the ARGOS survey (Freeman et al. 2013), and the HERBS survey (Duong et al. 2019a) and find that they are generally consistent. We report elemental abundances for $\mathrm{C}, \mathrm{Na}$, $\mathrm{Mg}, \mathrm{Al}, \mathrm{Si}, \mathrm{Ca}, \mathrm{Ti}, \mathrm{Cr}, \mathrm{Mn}, \mathrm{Fe}, \mathrm{Zn}, \mathrm{Ba}$, and $\mathrm{Ce}$. Using results from Lucey et al. (2020), which defines confined bulge stars as those with apocentres $<3.5 \mathrm{kpc}$, we divide our sample into five groups based on their dynamics. We associate these groups with different Galactic structures. Specifically, we label them as the inner bulge population, the outer bulge, the halo, and the thick and thin discs.

Given these data, we find the following evidence:

(i) The halo stars that pass through the inner Galaxy have relatively low chemical complexity compared to the inner and outer bulge populations. Specifically, the elemental abundances are highly correlated (mean correlation coefficient of 0.57 ) and have lower dimensionality (98.0 per cent of variance explained by four components) than the inner and outer bulge populations. This may indicate that these halo stars formed in situ in the disc and were heated to halo kinematics by a merger event (e.g. Di Matteo et al. 2019). However, it is also possible these stars formed ex situ in a single chemically simple progenitor or in many progenitors but the Universe, in general, was less chemically complex. The abundances for this halo population are consistent with a longer star formation time-scale when compared to the outer and inner bulge population.

(ii) The outer bulge population is very similar to the inner bulge population, although more chemically correlated (mean correlation coefficient of 0.53 ). The outer bulge is significantly distinct from the inner bulge stars in only its $\mathrm{Na}$ and $\mathrm{Mn}$ abundances, which both have metallicity-dependent yields in SNe II. Given that Na and Mn yields are higher in more metal-rich stars, it is likely the outer bulge stars were enriched by a more metal-rich population than the inner stars. This result is consistent with predictions from cosmological simulations that the outer bulge is younger than the inner bulge population (Tumlinson 2010).

(iii) The confined (or inner) bulge population is more chemically complex (mean correlation coefficient of 0.38 and 96.6 per cent of the variance explained by four components) than the unconfined stars in the bulge. Additionally, results from simulations predict that confined, or tightly bound, stars are generally older than unconfined, loosely bound stars (Tumlinson 2010). Combined, these results indicate that older populations in the inner Galaxy are generally more chemically complex than younger populations of similar metallicity. Furthermore, this suggests that the universe is more chemically complex early on, indicating either more diversity in chemical enrichment events or inhomogeneous mixing in the ISM.

(iv) We also find evidence that the $\mathrm{Ba}$ in low-metallicity $([\mathrm{Fe} / \mathrm{H}] \lesssim-1$ dex $)$ outer and inner bulge populations has similar origins to the $\alpha$-elements. Specifically, we find the $\mathrm{Ba}$ and $\alpha$ element abundances are positively correlated in these populations, but negatively correlated in the halo population.

(v) In our inner bulge population, we find one star that may show a signature of PISNe. Explicitly, this star has $[\mathrm{Fe} / \mathrm{H}]=-1.07 \mathrm{dex}$ and $[\mathrm{Ca} / \mathrm{Mg}]=0.83 \mathrm{dex}$, but its $[\mathrm{Al} / \mathrm{Mg}]$ ratio $(-0.30 \mathrm{dex})$ is higher and its $[\mathrm{Si} /(\mathrm{Mg}, \mathrm{Ca}, \mathrm{Fe})]$ ratio is lower than expected for PISN yields. Further observations are needed to measure more chemical abundances for this star and compare them to model PISN yields.

(vi) We detect two stars whose chemistry is consistent with second-generation globular cluster stars in that they show the signature $\mathrm{Mg}-\mathrm{Al}$ anticorrelation. One of these stars belongs to the inner bulge population while the other belongs to the outer bulge population. It is especially interesting that one star has an $[\mathrm{Al} / \mathrm{Mg}]$ ratio that is similar to what is observed for NGC 2808 that is theorized to be the core of the Gaia-Enceladus system (Myeong et al. 2018).

In total, this work demonstrates the power and necessity of combining chemistry with dynamics to disentangle and separately study the metal-poor stellar populations in the bulge region. In future work, we hope to achieve more precise dynamical parameters with improved astrometry from further Gaia data releases and the use of spectro-photometric distances. In addition, our work signifies the need for more measurements of neutron-capture elements in metal-poor bulge stars. However, further work on nucleosynthetic yields of $\mathrm{Ba}$ from Population II and III stars is needed to understand the correlation between $\mathrm{Ba}$ and $\alpha$-elements observed in this work. Furthermore, more precise photometry in the bulge is required to continue to target the most metal-poor bulge stars in large numbers with lower contamination rates from metal-rich stars.

\section{ACKNOWLEDGEMENTS}

This material is based on work supported by the National Science Foundation Graduate Research Fellowship under grant number 000392968. Victor P. Debattista is supported by Science and Tech- 
nology Facilities Council Consolidated grant \# ST/R000786/1. Thomas Bensby acknowledges financial support by grant number 2018-04857 from the Swedish Research Council. Keith Hawkins has been partially supported by a Time Domain Astrophysics/Scialog (2018-2020) grant funded by the Research Corporation and a Time Domain Astrophysics/Scialog grant (2019-2021) funded by the Heising-Simons Foundation. Madeline Lucey and Keith Hawkins acknowledge support from the National Science Foundation grant AST-1907417. Keith Hawkins is partially supported through the Wootton Center for Astrophysical Plasma Properties funded under the United States Department of Energy collaborative agreement DE-NA0003843. Chiaki Kobayashi acknowledges funding from the United Kingdom Science and Technology Facility Council (STFC) through grant ST/R000905/1.

This work has made use of data from the European Space Agency (ESA) mission Gaia (https://www.cosmos.esa.int/ gaia), processed by the Gaia Data Processing and Analysis Consortium (DPAC; https://www.cosmos.esa.int/web/gaia/dpac/ consortium). Funding for the DPAC has been provided by national institutions, in particular the institutions participating in the Gaia Multilateral Agreement.

This study is based on observations collected at the European Southern Observatory under ESO programme: 089.B-069.

\section{DATA AVAILABILITY}

The data underlying this article are available in the ESO Science Archive Facility at http://archive.eso.org/, and can be accessed with ESO programme ID 089.B-069.

\section{REFERENCES}

Adibekyan V. Z., Sousa S. G., Santos N. C., Delgado Mena E., González Hernández J. I., Israelian G., Mayor M., Khachatryan G., 2012, A\&A, $545, \mathrm{~A} 32$

Ahumada R. et al., 2020, ApJS, 249, 3

Allende Prieto C., 2004, Astron. Nachr., 325, 604

Allende Prieto C., Beers T. C., Wilhelm R., Newberg H. J., Rockosi C. M., Yanny B., Lee Y. S., 2006, ApJ, 636, 804

Allende Prieto C. et al., 2008, AJ, 136, 2070

Allende Prieto C., Hubeny I., Smith J. A., 2009, MNRAS, 396, 759

Alonso A., Arribas S., Martínez-Roger C., 1999, A\&AS, 140, 261

Amarsi A. M., Asplund M., 2017, MNRAS, 464, 264

Amarsi A. M., Asplund M., Collet R., Leenaarts J., 2016, MNRAS, 455, 3735

Arentsen A. et al., 2020a, MNRAS, 491, L11

Arentsen A. et al., 2020b, MNRAS, 496, 4964

Arentsen A. et al., 2021, MNRAS, 505, 1239

Armandroff T. E., Da Costa G. S., 1991, AJ, 101, 1329

Armandroff T. E., Zinn R., 1988, AJ, 96, 92

Babusiaux C. et al., 2010, A\&A, 519, A77

Barkat Z., Rakavy G., Sack N., 1967, Phys. Rev. Lett., 18, 379

Battaglia G., Irwin M., Tolstoy E., Hill V., Helmi A., Letarte B., Jablonka P., 2008, MNRAS, 383, 183

Battistini C., Bensby T., 2015, A\&A, 577, A9

Battistini C., Bensby T., 2016, A\&A, 586, A49

Beers T. C., Norris J. E., Placco V. M., Lee Y. S., Rossi S., Carollo D., Masseron T., 2014, ApJ, 794, 58

Bekki K., 2019, MNRAS, 490, 4007

Bensby T. et al., 2013, A\&A, 549, A147

Bensby T., Feltzing S., Oey M. S., 2014, A\&A, 562, A71

Bensby T. et al., 2017, A\&A, 605, A89

Bessell M. S., Castelli F., Plez B., 1998, A\&A, 333, 231

Blanco-Cuaresma S., Soubiran C., Heiter U., Jofré P., 2014, A\&A, 569, A111

Bournaud F., 2016, in Laurikainen E., Peletier R., Gadotti D., eds, Astrophysics and Space Science Library, Vol. 418, Galactic Bulges. Springer Int. Publ., Switzerland, p. 355
Bovy J., 2015, ApJS, 216, 29

Bromm V., 2013, Rep. Prog. Phys., 76, 112901

Brook C. B., Kawata D., Scannapieco E., Martel H., Gibson B. K., 2007, ApJ, 661, 10

Brown T. M. et al., 2010, ApJ, 725, L19

Buder S. et al., 2018, MNRAS, 478, 4513

Bureau M., Athanassoula E., 2005, ApJ, 626, 159

Calamida A. et al., 2014, ApJ, 790, 164

Carollo D. et al., 2019, ApJ, 887, 22

Carrillo A., Hawkins K., Bowler B. P., Cochran W., Vanderburg A., 2020, MNRAS, 491, 4365

Carroll J. A., 1933a, MNRAS, 93, 478

Carroll J. A., 1933b, MNRAS, 93, 680

Casagrande L., Wolf C., Mackey A. D., Nordland er T., Yong D., Bessell M., 2019, MNRAS, 482, 2770

Cescutti G., Chiappini C., 2014, A\&A, 565, A51

Cescutti G., Chiappini C., Hirschi R., Meynet G., Frischknecht U., 2013, A\&A, 553, A51

Cescutti G., Chiappini C., Hirschi R., 2018, in Chiappini C., Minchev I., Starkenburg E., Valentini M., eds, Proc. IAU Symp. 334, Rediscovering Our Galaxy. Kluwer, Dordrecht, p. 94

Choi J., Dotter A., Conroy C., Cantiello M., Paxton B., Johnson B. D., 2016, ApJ, 823, 102

Christlieb N., Schörck T., Frebel A., Beers T. C., Wisotzki L., Reimers D., 2008, A\&A, 484, 721

Clarkson W. I. et al., 2011, ApJ, 735, 37

Cole A. A., Smecker-Hane T. A., Tolstoy E., Bosler T. L., Gallagher J. S., 2004, MNRAS, 347, 367

Combes F., Sanders R. H., 1981, A\&A, 96, 164

Combes F., Debbasch F., Friedli D., Pfenniger D., 1990, A\&A, 233, 82

Cowan J. J., Thielemann F.-K., Truran J. W., 1991, Phys. Rep., 208, 267

Da Costa G. S. et al., 2019, MNRAS, 489, 5900

Debattista V. P., Mayer L., Carollo C. M., Moore B., Wadsley J., Quinn T., 2006, ApJ, 645, 209

Debattista V. P., Ness M., Gonzalez O. A., Freeman K., Zoccali M., Minniti D., 2017, MNRAS, 469, 1587

Dehnen W., 2000, AJ, 119, 800

Diemand J., Kuhlen M., Madau P., Zemp M., Moore B., Potter D., Stadel J., 2008, Nature, 454, 735

Di Matteo P., Haywood M., Lehnert M. D., Katz D., Khoperskov S., Snaith O. N., Gómez A., Robichon N., 2019, A\&A, 632, A4

Dotter A., 2016, ApJS, 222, 8

Duong L., Asplund M., Nataf D. M., Freeman K. C., Ness M., Howes L. M., 2019a, MNRAS, 486, 3586

Duong L., Asplund M., Nataf D. M., Freeman K. C., Ness M., 2019b, MNRAS, 486, 5349

El-Badry K., Rix H.-W., Ting Y.-S., Weisz D. R., Bergemann M., Cargile P., Conroy C., Eilers A.-C., 2018a, MNRAS, 473, 5043

El-Badry K. et al., 2018b, MNRAS, 480, 652

Fasano G., Franceschini A., 1987, MNRAS, 225, 155

Fernández-Trincado J. G. et al., 2017, ApJ, 846, L2

Frebel A. et al., 2006, ApJ, 652, 1585

Freeman K. et al., 2013, MNRAS, 428, 3660

Fulbright J. P., McWilliam A., Rich R. M., 2007, ApJ, 661, 1152

García Pérez A. E. et al., 2018, ApJ, 852, 91

Gilmore G. et al., 2012, The Messenger, 147, 25

Gonzalez O. A. et al., 2015, A\&A, 584, A46

Gratton R., Sneden C., Carretta E., 2004, ARA\&A, 42, 385

Grevesse N., Asplund M., Sauval A. J., 2007, Space Sci. Rev., 130, 105

Guedes J., Mayer L., Carollo M., Madau P., 2013, ApJ, 772, 36

Gustafsson B., Edvardsson B., Eriksson K., Jørgensen U. G., Nordlund Å., Plez B., 2008, A\&A, 486, 951

Hawkins K. et al., 2015, MNRAS, 447, 2046

Hawkins K. et al., 2016, A\&A, 592, A70

Heger A., Woosley S. E., 2010, ApJ, 724, 341

Heiter U., Jofré P., Gustafsson B., Korn A. J., Soubiran C., Thévenin F., 2015, A\&A, 582, A49, Paper I

Heiter U. et al., 2021, A\&A, 645, 63 
Hill V. et al., 2011, A\&A, 534, A80

Hirano S., Hosokawa T., Yoshida N., Omukai K., Yorke H. W., 2015, MNRAS, 448, 568

Horta D. et al., 2021, MNRAS, 500, 1385

Horta D. et al., 2021, MNRAS, 500, 5462

Howard C. D. et al., 2009, ApJ, 702, L153

Howes L. M. et al., 2014, MNRAS, 445, 4241

Howes L. M. et al., 2015, Nature, 527, 484

Howes L. M. et al., 2016, MNRAS, 460, 884

Iwamoto K., Brachwitz F., Nomoto K., Kishimoto N., Umeda H., Hix W. R., Thielemann F.-K., 1999, ApJS, 125, 439

Jofré P. et al., 2014, A\&A, 564, A133 Paper III

Johnson C. I., Rich R. M., Kobayashi C., Fulbright J. P., 2012, ApJ, 749, 175

Johnson C. I., Rich R. M., Kobayashi C., Kunder A., Pilachowski C. A., Koch A., de Propris R., 2013, ApJ, 765, 157

Johnson C. I., Rich R. M., Kobayashi C., Kunder A., Koch A., 2014, AJ, 148, 67

Johnson C. I. et al., 2020, MNRAS, 499, 2357

Karlsson T., Johnson J. L., Bromm V., 2008, ApJ, 679, 6

Kauffmann G., White S. D. M., Guiderdoni B., 1993, MNRAS, 264, 201

Keller S. C. et al., 2014, Nature, 506, 463

Kobayashi C., Nakasato N., 2011, ApJ, 729, 16

Kobayashi C., Umeda H., Nomoto K., Tominaga N., Ohkubo T., 2006, ApJ, 653,1145

Kobayashi C., Karakas A. I., Umeda H., 2011a, MNRAS, 414, 3231

Kobayashi C., Tominaga N., Nomoto K., 2011b, ApJ, 730, L14

Kobayashi C., Ishigaki M. N., Tominaga N., Nomoto K., 2014, ApJ, 785, L5

Kobayashi C., Karakas A. I., Lugaro M., 2020, ApJ, 900, 179

Koch A., Reichert M., Hansen C. J., Hampel M., Stancliffe R. J., Karakas A., Arcones A., 2019, A\&A, 622, A159

Kruijssen J. M. D., 2015, MNRAS, 454, 1658

Kuijken K., Rich R. M., 2002, AJ, 124, 2054

Kunder A. et al., 2016, ApJ, 821, L25

Kunder A. et al., 2020, AJ, 159, 270

Li T. S. et al., 2017, ApJ, 838, 8

Lind K., Asplund M., Barklem P. S., 2009, A\&A, 503, 541

Lind K., Asplund M., Barklem P. S., Belyaev A. K., 2011, A\&A, 528, A103

Lindegren L., 2018, Technical Report, Renormalising the Astrometric ChiSquare in Gaia DR2. Gaia DPAC

Lucey M. et al., 2019, MNRAS, 488, 2283

Lucey M. et al., 2020, MNRAS, 501, 5981

Marino A. F., Villanova S., Piotto G., Milone A. P., Momany Y., Bedin L. R., Medling A. M., 2008, A\&A, 490, 625

Mashonkina L. et al., 2008, A\&A, 478, 529

Masseron T., Merle T., Hawkins K., 2016, Astrophysics Source Code Library, record ascl: 1605.004

Mayor M. et al., 2003, The Messenger, 114, 20

McWilliam A., Rich R. M., 1994, ApJS, 91, 749

McWilliam A., Rich R. M., 2004, in McWilliam A., Rauch M., eds, Origin and Evolution of the Elements. Carnegie Observatories, Pasadena, p. 38

Merritt D., Sellwood J. A., 1994, ApJ, 425, 551

Monari G., Famaey B., Siebert A., Grand R. J. J., Kawata D., Boily C., 2016, MNRAS, 461, 3835

Myeong G. C., Evans N. W., Belokurov V., Sanders J. L., Koposov S. E., 2018, ApJ, 863, L28

Ness M., Freeman K., 2016, Publ. Astron. Soc. Aust., 33, e022

Ness M. et al., 2013a, MNRAS, 430, 836

Ness M. et al., 2013b, MNRAS, 432, 2092

Nishimura N., Takiwaki T., Thielemann F.-K., 2015, ApJ, 810, 109

Nomoto K., Kobayashi C., Tominaga N., 2013, ARA\&A, 51, 457

Nordlander T., Lind K., 2017, A\&A, 607, A75

Norris J. E., Christlieb N., Korn A. J., Eriksson K., Bessell M. S., Beers T. C., Wisotzki L., Reimers D., 2007, ApJ, 670, 774

Olszewski E. W., Schommer R. A., Suntzeff N. B., Harris H. C., 1991, AJ, 101,515

Ortolani S., Renzini A., Gilmozzi R., Marconi G., Barbuy B., Bica E., Rich R. M., 1995, Nature, 377, 701
Osorio Y., Barklem P. S., Lind K., Belyaev A. K., Spielfiedel A., Guitou M., Feautrier N., 2015, A\&A, 579, A53

Pancino E. et al., 2017, A\&A, 601, A112

Pasquini L. et al., 2002, The Messenger, 110, 1

Paxton B., Bildsten L., Dotter A., Herwig F., Lesaffre P., Timmes F., 2011, ApJS, 192, 3

Paxton B. et al., 2013, ApJS, 208, 4

Paxton B. et al., 2015, ApJS, 220, 15

Peacock J. A., 1983, MNRAS, 202, 615

Piskunov N., Valenti J. A., 2017, A\&A, 597, A16

Placco V. M., Frebel A., Beers T. C., Stancliffe R. J., 2014, ApJ, 797, 21

Portail M., Gerhard O., Wegg C., Ness M., 2017, MNRAS, 465, 1621

Quillen A. C., 2002, AJ, 124, 722

Quillen A. C., Minchev I., Sharma S., Qin Y.-J., Di Matteo P., 2014, MNRAS, 437,1284

Raha N., Sellwood J. A., James R. A., Kahn F. D., 1991, Nature, 352, 411

Rakavy G., Shaviv G., Zinamon Z., 1967, ApJ, 150, 131

Rich R. M., McWilliam A., 2000, in Bergeron J., ed., Proc. SPIE Conf. Ser. Vol. 4005, Discoveries and Research Prospects from 8- to 10-Meter-Class Telescopes. SPIE, Bellingham, p. 150

Roederer I. U., Preston G. W., Thompson I. B., Shectman S. A., Sneden C., Burley G. S., Kelson D. D., 2014, AJ, 147, 136

Rojas-Arriagada A. et al., 2014, A\&A, 569, A103

Rojas-Arriagada A. et al., 2017, A\&A, 601, A140

Rojas-Arriagada A. et al., 2020, MNRAS, 499, 1037

Rosswog S., Liebendörfer M., Thielemann F. K., Davies M. B., Benz W., Piran T., 1999, A\&A, 341, 499

Salvadori S., Ferrara A., Schneider R., Scannapieco E., Kawata D., 2010, MNRAS, 401, L5

Santistevan I. B., Wetzel A., El-Badry K., Bland-Hawthorn J., BoylanKolchin M., Bailin J., Faucher-Giguère C.-A., Benincasa S., 2020, MNRAS, 497, 747

Schiavon R. P. et al., 2017, MNRAS, 466, 1010

Sellwood J. A., Gerhard O., 2020, MNRAS, 495, 3175

Shapiro K. L., Genzel R., Förster Schreiber N. M., 2010, MNRAS, 403, L36

Shen J., Rich R. M., Kormendy J., Howard C. D., De Propris R., Kunder A., 2010, ApJ, 720, L72

Simmerer J., Sneden C., Cowan J. J., Collier J., Woolf V. M., Lawler J. E., 2004, ApJ, 617, 1091

Sitnova T. M., Yakovleva S. A., Belyaev A. K., Mashonkina L. I., 2020, Astron. Lett., 46, 120

Smiljanic R. et al., 2014, A\&A, 570, A122

Starkenburg E. et al., 2010, A\&A, 513, A34

Starkenburg E., Oman K. A., Navarro J. F., Crain R. A., Fattahi A., Frenk C. S., Sawala T., Schaye J., 2017a, MNRAS, 465, 2212

Starkenburg E. et al., 2017b, MNRAS, 471, 2587

Takahashi K., Yoshida T., Umeda H., 2018, ApJ, 857, 111

Truran J. W., 1981, A\&A, 97, 391

Tumlinson J., 2006, ApJ, 641, 1

Tumlinson J., 2010, ApJ, 708, 1398

Valenti J. A., Piskunov N., 1996, A\&AS, 118, 595

Valenti E., Zoccali M., Renzini A., Brown T. M., Gonzalez O. A., Minniti D., Debattista V. P., Mayer L., 2013, A\&A, 559, A98

Van der Swaelmen M., Hill V., Primas F., Cole A. A., 2013, A\&A, 560, A44

Vincenzo F., Kobayashi C., 2018, A\&A, 610, L16

Wegg C., Gerhard O., Portail M., 2015, MNRAS, 450, 4050

White S. D. M., Springel V., 2000, in Weiss A., Abel T. G., Hill V., eds, The First Stars. Springer-Verlag, Berlin, p. 327

Winteler C., Käppeli R., Perego A., Arcones A., Vasset N., Nishimura N., Liebendörfer M., Thielemann F. K., 2012, ApJ, 750, L22

Wolf C. et al., 2018, Publ. Astron. Soc. Aust., 35, e010

Woosley S. E., Heger A., 2006, ApJ, 637, 914

Woosley S. E., Weaver T. A., 1995, ApJS, 101, 181

Woosley S. E., Heger A., Weaver T. A., 2002, Rev. Mod. Phys., 74, 1015

Wylie S. M., Gerhard O. E., Ness M. K., Clarke J. P., Freeman K. C., BlandHawthorn J., 2021, A\&A, 653, A143 
Yong D. et al., 2013, ApJ, 762, 26

Zoccali M. et al., 2003, A\&A, 399, 931

Zoccali M., Hill V., Lecureur A., Barbuy B., Renzini A., Minniti D., Gómez A., Ortolani S., 2008, A\&A, 486, 177

Zoccali M. et al., 2014, A\&A, 562, A66

Zoccali M. et al., 2017, A\&A, 599, A12

\section{SUPPORTING INFORMATION}

Supplementary data are available at MNRAS online.

Please note: Oxford University Press is not responsible for the content or functionality of any supporting materials supplied by the authors.
Any queries (other than missing material) should be directed to the corresponding author for the article.

\section{APPENDIX A: ONLINE TABLE}

We show a section of the available online table in Table A1. The online table includes all 319 stars for which we measure stellar parameters and elemental abundances in this work and have derived orbital properties from COMBS II. In the table, we include all measured properties of the stars, including the SNRs, positions, velocities, dynamical properties, stellar parameters, and elemental abundances for every atomic line used in this work, along with the associated uncertainties.

Table A1. Stellar parameters, elemental abundances, and dynamical and observed properties.

\begin{tabular}{|c|c|c|c|c|c|c|c|c|c|c|c|}
\hline Object & $\begin{array}{c}l \\
(\mathrm{deg})\end{array}$ & $\begin{array}{c}b \\
(\operatorname{deg})\end{array}$ & $\begin{array}{c}\mathrm{SNR}_{06} \\
\left(\mathrm{pixel}^{-1}\right)\end{array}$ & $\begin{array}{l}\mathrm{T}_{\text {eff }} \\
(\mathrm{K})\end{array}$ & $\log g$ & {$[\mathrm{M} / \mathrm{H}]$} & {$[\mathrm{Fe} / \mathrm{H}]$} & $\mathrm{Fe}_{\text {scatter }}$ & {$[\mathrm{Mg} / \mathrm{H}]$} & $\mathrm{Mg}_{\text {scatter }}$ & ... \\
\hline 10078.0 & 15.45 & -9.67 & 74.81 & $4774 \pm 84$ & $1.92 \pm 0.3$ & $-1.61 \pm 0.17$ & $-1.29 \pm 0.04$ & 0.06 & $-1.36 \pm 0.12$ & 0.0 & $\ldots$ \\
\hline 10123.1 & 355.93 & -10.15 & 20.27 & $6137 \pm 127$ & $4.0 \pm 0.36$ & $-0.07 \pm 0.2$ & $0.16 \pm 0.18$ & 0.0 & $-0.15 \pm 0.17$ & 0.0 & $\ldots$ \\
\hline 1017.0 & 0.1 & -9.5 & 100.19 & $4921 \pm 83$ & $2.0 \pm 0.3$ & $-1.29 \pm 0.17$ & $-1.09 \pm 0.03$ & 0.07 & $-0.81 \pm 0.06$ & 0.01 & $\ldots$ \\
\hline 10205.0 & 15.45 & -9.64 & 39.35 & $4773 \pm 90$ & $2.75 \pm 0.31$ & $-1.12 \pm 0.18$ & $-1.31 \pm 0.09$ & 0.04 & $-0.74 \pm 0.07$ & 0.02 & $\ldots$ \\
\hline 1023.0 & 0.03 & -9.54 & 60.15 & $4946 \pm 84$ & $2.5 \pm 0.3$ & $-0.65 \pm 0.17$ & $-0.43 \pm 0.04$ & 0.03 & $-0.27 \pm 0.04$ & 0.05 & ... \\
\hline 10272.0 & 15.44 & -9.63 & 35.09 & $5422 \pm 94$ & $3.81 \pm 0.31$ & $-0.44 \pm 0.18$ & $-0.56 \pm 0.1$ & 0.01 & $-0.21 \pm 0.07$ & 0.0 & ... \\
\hline 10331.0 & 15.45 & -9.65 & 53.55 & $4769 \pm 85$ & $2.33 \pm 0.3$ & $-0.63 \pm 0.17$ & $-0.52 \pm 0.05$ & 0.08 & $-0.3 \pm 0.04$ & 0.08 & $\ldots$ \\
\hline 1036.0 & 0.06 & -9.53 & 61.26 & $5142 \pm 84$ & $2.25 \pm 0.3$ & $-0.96 \pm 0.17$ & $-0.5 \pm 0.06$ & 0.03 & $-0.52 \pm 0.1$ & 0.0 & ... \\
\hline 10397.0 & 15.47 & -9.66 & 23.7 & $4699 \pm 114$ & $3.72 \pm 0.34$ & $-0.53 \pm 0.19$ & $-0.82 \pm 0.08$ & 0.05 & $-0.23 \pm 0.14$ & 0.11 & ... \\
\hline 1070.0 & 359.93 & -9.61 & 54.5 & $4773 \pm 85$ & $2.18 \pm 0.3$ & $-1.43 \pm 0.17$ & $-1.43 \pm 0.07$ & 0.1 & $-1.12 \pm 0.07$ & 0.09 & $\ldots$ \\
\hline 1080.0 & 0.11 & -9.53 & 53.06 & $4660 \pm 85$ & $2.25 \pm 0.3$ & $-0.88 \pm 0.17$ & $-0.87 \pm 0.05$ & 0.01 & $-0.49 \pm 0.04$ & 0.05 & ... \\
\hline 10859.0 & 15.44 & -9.58 & 23.68 & $5176 \pm 114$ & $4.5 \pm 0.34$ & $-0.16 \pm 0.19$ & $-0.36 \pm 0.14$ & 0.0 & - & - & $\ldots$ \\
\hline 10896.0 & 15.51 & -9.72 & 37.13 & $4767 \pm 92$ & $2.5 \pm 0.31$ & $-1.22 \pm 0.18$ & $-1.33 \pm 0.1$ & 0.09 & $-0.82 \pm 0.09$ & 0.05 & $\ldots$ \\
\hline 1097.1 & 14.36 & -9.55 & 68.68 & $4908 \pm 84$ & $2.25 \pm 0.3$ & $-1.0 \pm 0.17$ & $-0.74 \pm 0.04$ & 0.02 & $-0.55 \pm 0.04$ & 0.06 & $\ldots$ \\
\hline 10985.0 & 15.48 & -9.64 & 22.03 & $6499 \pm 120$ & $3.85 \pm 0.35$ & $-0.28 \pm 0.19$ & - & - & - & - & $\ldots$ \\
\hline 11004.0 & 15.47 & -9.64 & 55.42 & $4347 \pm 85$ & $1.75 \pm 0.3$ & $-0.97 \pm 0.17$ & $-1.13 \pm 0.05$ & 0.06 & $-0.5 \pm 0.03$ & 0.01 & $\ldots$ \\
\hline 1106.0 & 359.83 & -9.67 & 85.36 & $4660 \pm 83$ & $1.71 \pm 0.3$ & $-1.9 \pm 0.17$ & $-1.85 \pm 0.05$ & 0.13 & - & - & $\ldots$ \\
\hline 1109.3 & 14.4 & -9.64 & 48.73 & $5192 \pm 86$ & $3.44 \pm 0.3$ & $0.33 \pm 0.17$ & $0.11 \pm 0.12$ & 0.15 & - & - & $\ldots$ \\
\hline 11151.0 & 15.55 & -9.77 & 26.0 & $5150 \pm 108$ & $3.19 \pm 0.33$ & $-0.13 \pm 0.19$ & $-0.16 \pm 0.1$ & 0.0 & $-0.04 \pm 0.07$ & 0.0 & $\ldots$ \\
\hline 1118.0 & 0.1 & -9.54 & 84.33 & $4449 \pm 83$ & $1.93 \pm 0.3$ & $-1.02 \pm 0.17$ & $-1.1 \pm 0.03$ & 0.08 & $-0.54 \pm 0.02$ & 0.02 & $\ldots$ \\
\hline 1129.0 & 359.84 & -9.67 & 38.71 & $4953 \pm 91$ & $2.75 \pm 0.31$ & $-1.02 \pm 0.18$ & $-1.0 \pm 0.12$ & 0.12 & $-0.6 \pm 0.13$ & 0.04 & $\ldots$ \\
\hline 1178.0 & 359.86 & -9.68 & 54.79 & $4462 \pm 85$ & $1.5 \pm 0.3$ & $-1.39 \pm 0.17$ & $-1.43 \pm 0.05$ & 0.09 & $-0.91 \pm 0.05$ & 0.02 & $\ldots$ \\
\hline 1189.0 & 0.18 & -9.52 & 32.26 & $5165 \pm 97$ & $2.25 \pm 0.32$ & $-1.08 \pm 0.18$ & $-0.69 \pm 0.09$ & 0.02 & $-0.78 \pm 0.12$ & 0.01 & $\ldots$ \\
\hline 1194.0 & 359.87 & -9.67 & 60.37 & $5282 \pm 84$ & $3.19 \pm 0.3$ & $-0.32 \pm 0.17$ & $-0.09 \pm 0.06$ & 0.09 & $-0.08 \pm 0.03$ & 0.0 & $\ldots$ \\
\hline 1196.2 & 355.73 & -10.23 & 33.14 & $5680 \pm 96$ & $3.57 \pm 0.31$ & $-0.36 \pm 0.18$ & $-0.34 \pm 0.22$ & 0.0 & $-0.32 \pm 0.21$ & 0.0 & $\ldots$ \\
\hline 11976.0 & 355.91 & -10.02 & 24.6 & $4350 \pm 111$ & $1.25 \pm 0.34$ & $-1.88 \pm 0.19$ & $-2.28 \pm 0.14$ & 0.1 & $-1.44 \pm 0.13$ & 0.0 & $\ldots$ \\
\hline 11985.1 & 14.33 & -9.7 & 24.29 & $4400 \pm 112$ & $1.64 \pm 0.34$ & $-1.36 \pm 0.19$ & $-1.41 \pm 0.24$ & 0.0 & $-1.06 \pm 0.16$ & 0.0 & $\ldots$ \\
\hline 1200.0 & 359.84 & -9.69 & 49.11 & $5909 \pm 86$ & $3.5 \pm 0.3$ & $-0.41 \pm 0.17$ & $-0.06 \pm 0.09$ & 0.05 & $-0.18 \pm 0.09$ & 0.0 & $\ldots$ \\
\hline 12063.3 & 14.28 & -9.59 & 33.32 & $4340 \pm 95$ & $1.25 \pm 0.31$ & $-1.42 \pm 0.18$ & $-1.5 \pm 0.09$ & 0.12 & $-0.94 \pm 0.07$ & 0.06 & $\ldots$ \\
\hline 1212.0 & 0.0 & -9.61 & 242.84 & $5040 \pm 83$ & $2.0 \pm 0.3$ & $-1.6 \pm 0.17$ & $-1.13 \pm 0.02$ & 0.03 & $-1.11 \pm 0.06$ & 0.03 & $\ldots$ \\
\hline 1214.0 & 360.0 & -9.62 & 77.14 & $4733 \pm 84$ & $2.42 \pm 0.3$ & $-0.58 \pm 0.17$ & $-0.67 \pm 0.04$ & 0.02 & $-0.28 \pm 0.04$ & 0.05 & ... \\
\hline 12311.3 & 14.28 & -9.63 & 90.9 & $4994 \pm 83$ & $3.25 \pm 0.3$ & $-0.85 \pm 0.17$ & $-0.95 \pm 0.04$ & 0.01 & $-0.46 \pm 0.03$ & 0.05 & $\ldots$ \\
\hline
\end{tabular}

Notes. A section of the online table with the object names, Galactic longitudes $(l)$ and latitudes $(b)$, the SNR of the HR06 spectra (SNR 06$)$, the effective temperature $\left(T_{\text {eff }}\right)$, surface gravity $(\log g)$, metallicity $([\mathrm{M} / \mathrm{H}]), \mathrm{Fe}$ abundance $([\mathrm{Fe} / \mathrm{H}])$, line-by-line scatter in the $\mathrm{Fe}$ abundance $\left(\mathrm{Fe}_{\text {scatter }}\right), \mathrm{Mg}$ abundance $([\mathrm{Mg} / \mathrm{H}])$, and the line-by-line scatter in the $\mathrm{Mg}$ abundance $\left(\mathrm{Mg} \mathrm{g}_{\text {scatter }}\right)$, Also included in the online table are the probability of confinement, distance, Galactic positions $(X, Y, Z)$, Galactic velocities $(U, V, W)$, eccentricity, apocentre, pericentre, $z_{\text {max }}$, and the $z$-component of the angular momentum $\left(L_{z}\right)$ along with the associated asymmetric uncertainties for all of these quantities. In addition, we include the $V$ sin $i$, right ascension, declination, Gaia source ID, ruwe, $G$-band magnitude, the RV (along with the associated uncertainty and scatter), the number of combined spectra, and the SNR for both the HR06 and HR21 spectra. In addition to the final mean $[\mathrm{X} / \mathrm{H}]$ abundance and associated uncertainty, we include all of the measured elemental abundances for each individual atomic line used, along with the associated uncertainty and the line-by-line scatter.

This paper has been typeset from a $\mathrm{T}_{\mathrm{E}} \mathrm{X} / \mathrm{LT} \mathrm{E} \mathrm{X}$ file prepared by the author. 